Pacific

Journal of

Mathematics

\title{
EQUIVARIANT ELLIPTIC GENERA
}

ROBERT WAELDER 


\title{
EQUIVARIANT ELLIPTIC GENERA
}

\author{
ROBERT WAELDER
}

\begin{abstract}
We introduce the equivariant elliptic genus for open varieties and prove an equivariant version of the change of variable formula for blow-ups along complete intersections. In addition, we prove the equivariant elliptic genus analogue of the McKay correspondence for the ALE spaces.
\end{abstract}

\section{Introduction}

The classical McKay correspondence describes a relationship between the representation theory of a finite subgroup $G \subset \mathrm{SU}(2)$ and the topology of the crepant resolution $\widehat{\mathbb{C}^{2} / G}$ of $\mathbb{C}^{2} / G$. One consequence of this relationship is that the Euler characteristic of $\widetilde{\mathbb{C}^{2} / G}$ is equal to the number of irreducible representations of $G$. A simple calculation shows that the number of irreducible representations of $G$ corresponds in turn to the orbifold Euler number of the pair $\left(\mathbb{C}^{2}, G\right)$. Here, if $X$ has an action by a finite group $G$, we define the orbifold Euler number $e_{\text {orb }}(X, G)=\frac{1}{|G|} \sum_{g h=h g} e\left(X^{g, h}\right)$, where $X^{g, h}$ denotes the common fixed point locus of a pair of commuting elements $g$ and $h$. This definition comes from string theory; in particular, physicists conjectured that, for $G$ a finite subgroup of $\mathrm{SU}(3)$, the orbifold Euler number of $\left(\mathbb{C}^{3}, G\right)$ coincided with the topological Euler number of a crepant resolution of the quotient, when such a resolution existed. In analogy with the classical McKay correspondence, we refer to formulae of this type as McKay correspondences for the Euler characteristic.

Investigations along these lines bring to mind several questions. First, what topological data should $e_{\mathrm{orb}}(X, G)$ correspond to when the quotient $X / G$ does not possess a crepant resolution? Second, what are the analogues of the McKay correspondence for other algebro-geometric invariants?

Batyrev [1999] used techniques from motivic integration to define the Euler number of a pair $(V, D)$, where $D$ is a divisor on $V$. The expression $e_{\mathrm{str}}(V, D)$ behaves well with respect to birational morphisms in the sense that $e_{\text {str }}(\widetilde{V}, \widetilde{D})=$ $e_{\text {str }}(V, D)$ if $K_{\widetilde{V}}+\widetilde{D}=\phi^{*}\left(K_{V}+D\right)$ for a birational morphism $\phi: \widetilde{V} \rightarrow V$. This definition therefore provides a framework for studying the Euler number of a resolution of singularities even when no crepant resolution exists. For a special

MSC2000: primary 14E99; secondary 57R91.

Keywords: elliptic genus, equivariant cohomology, McKay correspondence, rigidity. 
choice of a divisor $\Delta$ on $X / G$, Batyrev proved that $e_{\mathrm{orb}}(X, G)=e_{\mathrm{str}}(X / G, \Delta)$. In fact, he proved a much stronger variation of this theorem for the $\chi_{y}$ genus.

An important generalization of both the topological Euler characteristic and the $\chi_{y}$ genus is the two variable elliptic genus. If $X$ is an almost complex manifold, the elliptic genus $E l l(X)$ is defined as

$$
\int_{X} \prod_{T X} \frac{x_{j} \theta\left(\frac{x_{j}}{2 \pi i}-z, \tau\right)}{\theta\left(\frac{x_{j}}{2 \pi i}, \tau\right)} .
$$

The product is taken over the formal Chern roots of the holomorphic tangent bundle to $X . \theta(t, \tau)$ is the Jacobi theta function and $z$ is a formal parameter.

When $X$ possesses an action of a finite group $G$, there exists a notion of the orbifold elliptic genus of $X$ which extends Batyrev's definition of the orbifold $\chi_{y}$ genus. Recently, Borisov and Libgober [2005] proved the elliptic genus analogue of the McKay correspondence. To do this, they first define the elliptic genus and orbifold elliptic genus of a pair $(X, D)$ for $D$ a divisor on $X$ and show that these definitions satisfy change of variable formulae similar to the objects $e_{\text {str }}(X, D)$ in Batyrev's paper. Whereas Batyrev's proof relies on the change of variables formula from motivic integration, Borisov and Libgober examine the case of a single blowup and appeal to the deep result of Włodarczyk [2003] that every birational map of smooth complex varieties may be factored into a sequence of blow-ups and blowdowns along smooth centers. This change of variable formula allowed Borisov and Libgober to reduce the proof to a version of the McKay correspondence for toroidal morphisms. A crucial aspect of their proof is a description of the cohomological pushforward of a toroidal morphism in terms of combinatorial data associated to the map. We refer to this technique as Borisov and Libgober's push-forward formula.

When $X$ has an action of a compact torus $T$ that commutes with the action of a finite group $G$, one has natural definitions for the equivariant orbifold elliptic genus of $(X, G)$ and the equivariant elliptic genus of $T$-resolutions of $X / G$. One reason for studying these equivariant elliptic genera is that, by localization, they make sense even when $X$ is not compact, provided that $X$ has compact fixed components. Here we prove an equivariant elliptic genus analogue of the classical McKay correspondence for ALE spaces. Along the way we prove the equivariant version of the change of variable formula for blow-ups along complete intersections. In the toric case, this formula turns out to be linked to a rigidity property of the elliptic genus of a pair. A prominent feature throughout this paper is the equivariant analogue of Borisov and Libgober's push-forward formula; we describe a relationship between their formula and the functorial localization formula for a toric morphism. The suggestion that Borisov and Libgober's push-forward formula is really functorial localization in disguise might explain the ease with which the proof of their formula extends to the equivariant case. 
Here is an outline of the paper. In Section 2 we introduce the notion of the equivariant orbifold elliptic class, which is useful for making sense of elliptic genera on open varieties with a torus action. In Section 3 we discuss various aspects of equivariant cohomology and prove some technical lemmas which will be used implicitly throughout the paper. In Sections 4 and 5 we prove the equivariant analogue of Borisov and Libgober's push-forward formula, and discuss its relationship to the functorial localization formula applied to a toric morphism. In Section 6 we prove a rigidity theorem for the elliptic genus of a toric pair $(X, D)$ and discuss its relationship to the change of variable formula, which we prove in Theorem 7.1. In Section 8 we use this results to prove an equivariant elliptic genus analogue of the McKay correspondence for ALE spaces. Finally, in Section 9 we discuss the relationship between the equivariant elliptic genus and Batyrev's stringy Euler number.

\section{Equivariant orbifold elliptic class}

Let $X^{n}$ be a smooth compact variety and $D=\sum_{i} \delta_{i} D_{i}$ a smooth normal crossing divisor, with coefficients $\delta_{i}<1$. Let $G$ be a finite group acting holomorphically on $X$. For $g, h \in G$ a commuting pair, let $\left\{X_{\gamma}^{g, h}\right\}$ denote the connected components of their common fixed point locus. Fix one such component $X_{\gamma}^{g, h}$. The normal bundle $N_{X_{\gamma}^{g, h}}$ splits as a sum $\bigoplus_{\lambda} N_{\lambda}$ over irreducible characters for the subgroup $(g, h)$. For $x \in(g, h)$, let $\lambda(x) \in \mathbb{Q} \cap[0,1)$ be the rational number such that $x$ acts on the fibers of $N_{\lambda}$ as multiplication by $e^{2 \pi i \lambda(x)}$.

Now fix an irreducible component $D_{i}$ of $D$. If $X_{\gamma}^{g, h}$ is contained in $D_{i}$ then

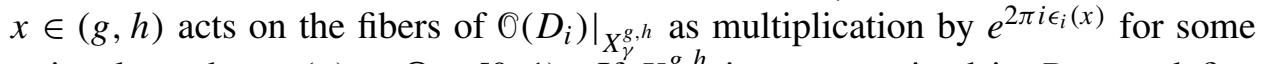
rational number $\epsilon_{i}(x) \in \mathbb{Q} \cap[0,1)$. If $X_{\gamma}^{g, h}$ is not contained in $D_{i}$, we define $\epsilon_{i}=0$. Of course the functions $\lambda$ and $\epsilon_{i}$ depend on the choice of the commuting pair $(g, h)$ and on the connected component $X_{\gamma}^{g, h}$ of $X^{g, h}$. We will omit making explicit reference to this dependence in order to simplify the notation.

Following [Borisov and Libgober 2005], we define the orbifold elliptic genus of the pair $(X, D)$ by the formula

$$
\begin{aligned}
E l l_{\mathrm{orb}}(X, D, G)= & \frac{1}{|G|} \sum_{g h=h g, \gamma} \int_{X_{\gamma}^{g, h}} \prod_{T X_{\gamma}^{g, h}} \frac{x_{j} \theta\left(\frac{x_{j}}{2 \pi i}-z\right)}{\theta\left(\frac{x_{j}}{2 \pi i}\right)} \\
& \times \prod_{N_{\lambda}} \frac{\theta\left(\frac{x_{\lambda}}{2 \pi i}+\lambda(g)-\lambda(h) \tau-z\right)}{\theta\left(\frac{x_{\lambda}}{2 \pi i}+\lambda(g)-\lambda(h) \tau\right)} e^{2 \pi i \lambda(h) z} \\
& \times \prod_{D_{i}} \frac{\theta\left(\frac{D_{i}}{2 \pi i}+\epsilon_{i}(g)-\epsilon_{i}(h) \tau-\left(-\delta_{i}+1\right) z\right) \theta(-z)}{\theta\left(\frac{D_{i}}{2 \pi i}+\epsilon_{i}(g)-\epsilon_{i}(h) \tau-z\right) \theta\left(-\left(-\delta_{i}+1\right) z\right)} e^{-2 \pi i \delta_{i} \epsilon_{i}(h) z} .
\end{aligned}
$$


Here of course we mean by $x_{j}$ the Chern roots of $T X_{\gamma}^{g, h}$, by $x_{\lambda}$ the Chern roots of $N_{\lambda}$, and by $D_{i}$ the first Chern classes of the corresponding divisors.

Now assume that $X$ has a $T$-action which commutes with the action of $G$, and that the irreducible components of $D$ are $T$-invariant. Assume that the action lifts to the bundles $O\left(D_{i}\right)$. We define the equivariant orbifold elliptic genus $E l l_{\text {orb }}^{T}$ as follows: For each fixed component $P \subset X_{\gamma}^{g, h}$, let $v_{j} \in \mathfrak{t}^{*}$ denote the infinitesimal weights of the torus action on the fibers of the normal bundle $v_{P / X_{\gamma}^{g, h}}$. Similarly, let $\chi_{\lambda} \in \mathfrak{t}^{*}$ denote the infinitesimal weights of the torus action on $\left.N_{X_{\gamma}^{g, h}}\right|_{P}$. If $P \subset D_{i}$, let $\eta_{i}$ denote the infinitesimal weight of the torus action on $\left.\mathcal{O}\left(D_{i}\right)\right|_{P}$. Otherwise, let $\eta_{i}=0$. All of the above weights depend on the fixed component $P$ and on the commuting pair $g, h$. Again, we leave this dependence out of the notation in order to avoid cluttering. With this in mind, we define $\operatorname{Ell}_{\text {orb }}^{T}(X, D, G)$ to be

$$
\begin{aligned}
\frac{1}{|G|} \sum_{g h=h g, \gamma} \sum_{P \subset X_{\gamma}^{g, h}} \int_{P} \prod_{T P} \frac{p_{k} \theta\left(\frac{p_{k}}{2 \pi i}-z\right)}{\theta\left(\frac{p_{k}}{2 \pi i}\right)} \prod_{v_{P}} \frac{\theta\left(\frac{n_{j}}{2 \pi i}+v_{j}-z\right)}{\theta\left(\frac{n_{j}}{2 \pi i}+v_{j}\right)} \\
\times \prod_{N_{\lambda}} \frac{\theta\left(\frac{x_{\lambda}}{2 \pi i}+\chi_{\lambda}+\lambda(g)-\lambda(h) \tau-z\right)}{\theta\left(\frac{x_{\lambda}}{2 \pi i}+\chi_{\lambda}+\lambda(g)-\lambda(h) \tau\right)} e^{2 \pi i \lambda(h) z} \\
\times \prod_{D_{i}} \frac{\theta\left(\frac{D_{i}}{2 \pi i}+\eta_{i}+\epsilon_{i}(g)-\epsilon_{i}(h) \tau-\left(-\delta_{i}+1\right) z\right) \theta(-z)}{\theta\left(\frac{D_{i}}{2 \pi i}+\eta_{i}+\epsilon_{i}(g)-\epsilon_{i}(h) \tau-z\right) \theta\left(-\left(-\delta_{i}+1\right) z\right)} e^{-2 \pi i \delta_{i} \epsilon_{i}(h) z} .
\end{aligned}
$$

Finally, motivated by [Borisov and Libgober 2005], we introduce the notion of the equivariant elliptic class

$$
\mathscr{E} l l_{\mathrm{orb}}^{T}(X, D, G) \in H_{T}^{*}(X) .
$$

For convenience, assume that every component $X_{\gamma}^{g, h}$ of $X^{g, h}$ is a connected component of $D_{i_{1}} \cap \cdots \cap D_{i_{r}}$ for some indexing set $I_{\gamma}^{g, h}=\left\{i_{k}\right\}$. We also assume that $D$ is $G$-normal. Then $T X-\bigoplus_{k=1}^{r} \mathcal{O}\left(D_{i_{k}}\right) \in K_{T}(X)$ is a bundle which equals $T X_{\gamma}^{g, h}$ when restricted to $X_{\gamma}^{g, h}$. Thus, consider the class

$$
\begin{array}{r}
\left(\frac{2 \pi i \theta(-z)}{\theta^{\prime}(0)}\right)^{n-r} \Phi_{X_{\gamma}^{g, h}}^{T} \prod_{T X} \frac{\frac{x_{j}(t)}{2 \pi i} \theta\left(\frac{x_{j}(t)}{2 \pi i}-z\right) \theta^{\prime}(0)}{\theta\left(\frac{x_{j}(t)}{2 \pi i}\right) \theta(-z)} \prod_{I_{\gamma}^{g, h} \frac{}{D_{i}(t)} \frac{\theta\left(\frac{D_{i}(t)}{2 \pi i}\right) \theta\left(\frac{D_{i}(t)}{2 \pi i}-z\right) \theta^{\prime}(0)}{2 \pi i}} \\
\times \prod_{I_{\gamma}^{g, h}} \frac{\theta\left(\frac{D_{i}(t)}{2 \pi i}+\epsilon_{i}(g)-\epsilon_{i}(h) \tau-\left(-\delta_{i}+1\right) z\right) \theta(-z)}{\theta\left(\frac{D_{i}(t)}{2 \pi i}+\epsilon_{i}(g)-\epsilon_{i}(h) \tau\right) \theta\left(-\left(-\delta_{i}+1\right) z\right)} e^{2 \pi i\left(-\delta_{i}+1\right) \epsilon_{i}(h) z} \\
\times \prod_{I_{X}-I_{\gamma}^{g, h}} \frac{\theta\left(\frac{D_{k}(t)}{2 \pi i}-\left(-\delta_{k}+1\right) z\right) \theta(-z)}{\theta\left(\frac{D_{k}(t)}{2 \pi i}-z\right) \theta\left(-\left(-\delta_{k}+1\right) z\right)} .
\end{array}
$$


Here the parameter $t$ in expressions such as $x_{j}(t)$ and $D_{k}(t)$ refers to the equivariant Chern roots. When $X$ is simply connected we can always make sense of this definition (see Section 3.2). The term $\Phi_{X_{\gamma}^{g, h}}^{T}$ is the equivariant Thom class of $X_{\gamma}^{g, h}$ in $X$. All of the fractions $\epsilon_{i}$ implicitly depend on $\gamma$. By localization, the integral of this class over $X$ is the contribution from $X_{\gamma}^{g, h}$ to the equivariant orbifold elliptic genus. We call this equivariant cohomology class $\mathscr{E} l l_{\text {orb }}^{T}\left(D, X_{\gamma}^{g, h}\right)$. We define the equivariant orbifold elliptic class

$$
\mathscr{E} l l_{\mathrm{orb}}^{T}(X, D, G)=\frac{1}{|G|} \sum_{g h=h g, \gamma} \mathscr{E} l l_{\mathrm{orb}}^{T}\left(D, X_{\gamma}^{g, h}\right) .
$$

When $G=1$, this is called simply the equivariant elliptic class $\mathscr{E} l l(X, D)$. We call the integral of this class the equivariant elliptic genus $\operatorname{Ell}_{T}(X, D)$.

Suppose that $f: \widetilde{X} \rightarrow X$ is the blow-up of $X$ along a $T \times G$-invariant subvariety. Define $\widetilde{D}$ on $\widetilde{X}$ so that $f^{*}\left(K_{X}+D\right)=K_{\widetilde{X}}+\widetilde{D}$.

$$
\text { Theorem 2.1. } \quad f_{*} \mathscr{E} l l_{\text {orb }}^{T}(\widetilde{D}, \widetilde{X}, G)=\mathscr{E} l l_{\text {orb }}^{T}(D, X, G) \text {. }
$$

This is the equivariant analogue of the change of variable formulae discovered by Chin-Lung Wang and Borisov and Libgober. We will refer to the above formula as the change of variable formula for the orbifold elliptic class. We refer to the formula obtained by integrating both sides as the change of variable formula for the orbifold elliptic genus. For a proof of the formula in this general case, see [Waelder 2007]. For our present purposes, we will only need to examine the simpler situation in which the blow-up locus and orbifold fixed data of $X$ are complete intersections. For that case, we will provide a complete proof in Theorem 7.1.

The value of the change of variable formula is that it allows us to compare orbifold elliptic data between varieties which are birationally equivalent. In Section 8 we will provide an interesting application of the change of variable formula to the computation of equivariant elliptic indices of ALE spaces. The approach we take is inspired by Borisov and Libgober's proof of the nonequivariant McKay correspondence for the elliptic genus.

Remark 2.2. If $X$ has a torus action with compact fixed components, via the localization formula we can always make sense of the quantity $\operatorname{Ell}_{\mathrm{orb}}^{T}(X, D, G)$ even when $X$ is open. We will continue to refer to this quantity as the equivariant orbifold elliptic genus of $X$.

Remark 2.3. A word on notation: There are many objects associated to a variety $X$ which encode the data of the equivariant elliptic genus of $X$. We use the prefix $E l l_{T}$ to refer to objects in $H_{T}^{*}(p t), \mathscr{E} l l_{T}$ to refer to objects in $H_{T}^{*}(X)$, and $\mathscr{E} \mathscr{L} \mathscr{L}_{T}$ to refer to objects in $K_{T}(X)$. 


\section{Preliminaries on equivariant cohomology}

In this section we gather the ingredients from equivariant cohomology which we will be using in this paper. For a thorough reference on the subject, see [Atiyah and Bott 1984].

3.1. Definitions and localization. Let $X$ be a smooth $T$-space, where $T$ is a compact torus of rank $\ell$. Let $E T=\left(S^{\infty}\right)^{\ell}$. ET is a contractible space on which $T$ acts freely. The diagonal action of $T$ on $X \times E T$ therefore gives rise to a smooth (infinite-dimensional) quotient $X_{T}=(X \times E T) / T$. It is easy to see that $X_{T}$ is a fiber bundle over $B T=E T / T$ with fiber $X$. Define the equivariant cohomology group $H_{T}^{*}(X)=H^{*}\left(X_{T}\right)$.

The translation of concepts from cohomology to equivariant cohomology is more or less routine. For example, a $T$-map $f: X \rightarrow Y$ gives rise to a natural map $f_{T}: X_{T} \rightarrow Y_{T}$, and therefore induces a pullback $f^{*}: H_{T}^{*}(Y) \rightarrow H_{T}^{*}(X)$. Similarly, for any $E \in K_{T}(X), E_{T}$ defines a finite rank vector bundle over $X_{T}$ which corresponds to the vector bundle $E \rightarrow X$ over every fiber of $X_{T} \rightarrow B T$. In this way, we may define the equivariant characteristic classes of $E$ to be the characteristic classes of $E_{T}$.

If $p$ is a single point with trivial $T$-action, the equivariant map $\pi: X \rightarrow p$ induces a map $\pi^{*}: H_{T}^{*}(p) \rightarrow H_{T}^{*}(X)$. Since $H_{T}^{*}(p)=H^{*}(B T)=\mathbb{C}\left[u_{1}, \ldots, u_{\ell}\right]$, the map $\pi^{*}$ makes $H_{T}^{*}(X)$ into a $\mathbb{C}\left[u_{1}, \ldots, u_{\ell}\right]$-module. Define $H_{T}^{*}(X)_{\text {loc }}=H_{T}^{*}(X) \otimes_{\mathbb{C}\left[u_{1}, \ldots, u_{\ell}\right]}$ $\mathbb{C}\left(u_{1}, \ldots, u_{\ell}\right)$. A fundamental result of the subject is the localization theorem:

Theorem 3.1. Let $\{P\}$ denote the set of $T$-fixed components of $X$. Then $H_{T}^{*}(X)_{\mathrm{loc}} \cong$ $\bigoplus_{P} H^{*}(P) \otimes \mathbb{C}\left(u_{1}, \ldots, u_{\ell}\right)$.

If $P$ is a fixed component of $X$, the normal bundle to $P$ splits as a sum over the characters of the $T$-action on the fibers: $N_{P}=\bigoplus_{\lambda} V_{\lambda}$. Let $n_{\lambda}^{i}$ denote the formal Chern roots of $V_{\lambda}$. If we identify the equivariant parameters $u_{1}, \ldots, u_{\ell}$ with linear forms on the Lie algebra of $T$, then the equivariant Euler class $e(P)$ of $N_{P}$ is equal to $\prod_{\lambda} \prod_{i}\left(n_{\lambda}^{i}+\lambda\right)$. Since none of the characters $\lambda$ are equal to zero, we see that $e(P)$ is always invertible. In light of this fact, we can describe the above isomorphism more explicitly. The map $H_{T}^{*}(X)_{\mathrm{loc}} \rightarrow \bigoplus_{P} H^{*}(P) \otimes \mathbb{C}\left(u_{1}, \ldots, u_{\ell}\right)$ is given by $\omega \mapsto \bigoplus_{P} i_{P}^{*} \omega / e(P)$, where $i_{P}: P \hookrightarrow X$ is the inclusion map.

If $f: X \rightarrow Y$ is a proper map of $T$-spaces, we have the equivariant analogue of the cohomological push-forward $f_{*}: H_{T}^{*}(X) \rightarrow H_{T}^{*}(Y)$. As in the nonequivariant setting, $f_{*}$ satisfies the projection formula $f_{*}\left(f^{*}(\omega) \wedge \eta\right)=\omega \wedge f_{*} \eta$. The new feature in equivariant cohomology is that we have an explicit expression for the restriction of $f_{*} \omega$ to a fixed component in $Y$. This is given by the functorial localization formula [Lian et al. 1999a; 1999b]: 
Theorem 3.2. Let $f: X \rightarrow Y$ be a proper map of T-spaces. Let $P$ be a fixed component of $Y$ and let $\{F\}$ be the collection of fixed components in $X$ which $f$ maps into $P$. Let $\omega \in H_{T}^{*}(X)$. Then

$$
\sum_{F} f_{*} \frac{i_{F}^{*} \omega}{e(F)}=\frac{i_{P}^{*} f_{*} \omega}{e(P)} .
$$

Suppose $f: X \rightarrow Y$ is a proper map of $n$-dimensional $T$ spaces with isolated fixed points. For $F$ a fixed point in $X$, let $\lambda(F)_{1}+\cdots+\lambda(F)_{n}$ denote the decomposition of $T_{F} X$ into irreducible characters. Clearly $e(F)=\prod_{j=1}^{n} \lambda(F)_{j}$. Moreover, each $\omega \in H_{T}^{*}(X)$ is defined by a collection of polynomial functions (with relations) $\omega_{F} \in \mathbb{C}\left[u_{1}, \ldots, u_{\ell}\right]$ attached to the fixed points $F$ in $X$. For $P$ a fixed point in $Y$, we have, by functorial localization,

$$
\left(f_{*} \omega\right)_{P}=\sum_{F} \omega_{F} \prod_{j=1}^{n} \frac{\lambda(P)_{j}}{\lambda(F)_{j}} .
$$

In the next two sections we will discuss the similarity between this formula and the push-forward formula of Borisov and Libgober.

Note that the localization techniques discussed here continue to hold in the ring formed by uniformly convergent power series of equivariant classes, which is more precisely the domain of definition for the equivariant elliptic class. For simplicity of exposition, we will not make that distinction here; but see [Waelder 2007] for a discussion of this technical point.

Before ending this subsection, we make one final remark on an alternative approach to equivariant cohomology. Let $e_{1}, \ldots, e_{\ell}$ form a basis for the Lie algebra of $T$ which is dual to the linear forms $u_{1}, \ldots, u_{\ell}$. Every $V \in \mathfrak{t}$ defines a vector field $V$ on $X$ by the formula

$$
V(p)=\left.\frac{d}{d t}\right|_{t=0} \exp (t V) \cdot p
$$

Define $\Omega_{T}^{*}(X)$ to be the ring of differential forms on $X$ which are annihilated by $\mathscr{L}_{V}$ for every $V \in \mathfrak{t}$. If we let $d_{\mathfrak{t}}=d+\sum_{\alpha=1}^{\ell} u_{\alpha} i_{e_{\alpha}}$, then $d_{\mathfrak{t}}$ defines an operator on $\Omega_{T}^{*}(X) \otimes \mathbb{C}\left[u_{1}, \ldots, u_{\ell}\right]$ and satisfies $d_{\mathfrak{t}}^{2}=0$. The Cartan model for equivariant cohomology is defined to be

$$
H_{T}^{*}(X)_{\text {Cartan }}=\frac{\operatorname{ker} d_{\mathfrak{t}}}{\operatorname{im} d_{\mathfrak{t}}}
$$

It is well known that $H_{T}^{*}(X)_{\text {Cartan }} \cong H_{T}^{*}(X)$. See [Atiyah and Bott 1984] for details. Throughout, we will switch freely between the two descriptions. 
3.2. Equivariant Chern class of a divisor. Let $X$ be a smooth compact simply connected complex manifold with a $T$-action. For simplicity of notation, assume that $T=S^{1}$. We also omit the equivariant parameters in this section, since they clutter the notation and play no role in the proofs. Let $D \subset X$ be a $T$-invariant irreducible Cartier divisor with associated line bundle $O(D)$. Let $\omega$ be a representative of the Thom class of the normal bundle, $N_{D}$, of $D$. By averaging over $T$, we may assume that $\omega$ is $T$-invariant.

Let $V$ be the vector field on $X$ induced by the $T$-action. We are presented with two natural procedures for extending $\omega$ to an equivariant cohomology class, i.e., a $T$-invariant class in the kernel of $d+i_{V}$. First, since $\omega$ is invariant and closed, $\mathscr{L}_{V} \omega=d i_{V} \omega=0$, so $i_{V} \omega$ defines a class in $H^{1}(X)$. Since $X$ is simply connected, $i_{V} \omega=d f$. If we require that $f$ have compact support in $N_{D}$, then the above moment map equation defines $f$ uniquely, and $\omega-f$ defines an equivariant extension of $\omega$. Second, since $X$ is simply connected and $T$ is abelian, we may lift the action of $T$ to $O(D)$. The equivariant first Chern class of $O(D)$ then defines another equivariant extension of $\omega$.

In this section, we show that both extensions represent the same equivariant class provided we choose an "appropriate" lift of the action of $T$ to $O(D)$. By appropriate, we mean that the action of $T$ on $\mathcal{O}(D)$ extends the natural action of $T$ on $\left.O(D)\right|_{p}$ for any fixed point $p$. Note that for dimensionality reasons, the two equivariant extensions can differ by at most a constant. The goal in this section is to prove that this constant is zero.

Lemma 3.3. Let $\omega$ and $f$ be defined as above. Let $p \in D$ be a fixed point of the $T$ action and let $a=a(p)$ be the infinitesimal weight of the character $\left.O(D)\right|_{p}$. Then $f(p)=-a$.

Proof. Let $U=\left\{\left(z_{1}, \ldots, z_{n}\right)\right\}$ be a coordinate system centered at $p$, with $D$ defined by $\left\{z_{n}=0\right\}$. We can choose this coordinate system so that $e^{i t} \cdot\left(z_{1}, \ldots, z_{n}\right)=$ $\left(e^{i m_{1} t} z_{1}, \ldots, e^{i m_{n-1} t} z_{n-1}, e^{i a t} z_{n}\right)$. Let $d \theta$ be the $T$-invariant angular form in the $z_{n}$-coordinate plane. Call this plane $U_{n}$. Let $r$ be the distance function on $U_{n}$ and $\rho(r)$ a bump function which integrates to 1 over $U_{n}$ and is identically equal to 1 in a neighborhood of the origin. Then the Thom class corresponding to the hyperplane $z_{n}=0$ is represented by the $T$-invariant form $d(\rho(r) d \theta)$ in this neighborhood. It follows that $\left.\omega\right|_{U}=d(\rho(r) d \theta)+d \psi$, where $\psi$ is a form with compact support in the $z_{n}$-direction. Since all the forms involved are $T$-invariant, we may assume that $\psi$ is $T$-invariant. In this coordinate system, the vector field $V$ takes the form $a \frac{\partial}{\partial \theta}$ in the $U_{n}$ plane. Thus $i_{V} d(\rho d \theta)=-d\left(i_{V} \rho d \theta\right)=-d(a \rho(r))$. We therefore have that $i_{V} \omega=-d(a \rho)-d\left(i_{V} \psi\right)$. Since $a \rho+i_{V} \psi$ have compact support in the vertical direction and satisfy $d\left(a \rho+i_{V} \psi\right)=-d f$, we must have $f=-a \rho-i_{V} \psi$. Since $V(p)=0$, this implies that $f(p)=-a$. 
We next prove that we can always adjust the action of $T$ on $\mathcal{O}(D)$ so that it coincides with the natural action of $\left.\mathcal{O}(D)\right|_{p}$ for any fixed point $p \in D$. The ensuing discussion follows closely the ideas of section 8 of [Atiyah and Bott 1984]. Let $\nabla$ be a $T$-invariant connection on $\mathcal{O}(D)$ with corresponding connection 1-form $\theta$. View the vector field $V$ as an operator acting on $\Gamma(X, O(D))$. If $s$ is a local frame, then $V s=L(s) s$ for some smooth function $L$ which depends on $s$. The statement that $\nabla$ is $T$-invariant means that $\nabla V=V \nabla$. Fix a local frame $s$ satisfying $d s=0$ in local coordinates. Then $\nabla V s=\nabla L s=d L s+L \theta s$ and $V \nabla s=V \theta s=\mathscr{L}_{V} \theta s+\theta L s$. It follows that

$$
d L(s)=\mathscr{L}_{V} \theta=i_{V} d \theta+d i_{V} \theta .
$$

Now $d \theta=-2 \pi i \omega+d \eta$ for some $T$-invariant 1 -form $\eta$. Thus, $i_{V} d \theta=-2 \pi i d f-$ $d\left(i_{V} \eta\right)$. Thus, the above equation implies $L(s)=-2 \pi i f+i_{V} \theta-i_{V} \eta+2 \pi i c$, where $c$ is a constant. It is easy to check that this constant is independent of the section $s$. It follows that the infinitesimal action of $T$ on $\left.O(D)\right|_{p}$ is given by $-2 \pi i f(p)+2 \pi i c$. Thus, the infinitesimal weight attached to every fixed point $p$ is $a(p)+c$. If $c \neq 0$, we can replace $\mathcal{O}(D)$ with $O(D) \otimes \mathcal{O}_{c}$, where the action of $T$ on $O_{c}$ takes the global section 1 to $e^{-2 \pi i c}$. Thus, we have proven that we can always lift the action of $T$ so that it coincides with the natural action on $\left.\mathcal{O}(D)\right|_{p}$ for fixed points $p$. Whenever we speak of $O(D)$ as an equivariant bundle, we will assume this choice of a lifted action.

Finally, we prove that the equivariant first Chern class of $O(D)$ coincides with $\omega-f$. By localization, it suffices to prove that $c_{1}^{T}(\mathcal{O}(D))=\omega-f$ at every fixed point. But this follows from the well-known observation that $\left.c_{1}^{T}(\mathcal{O}(D))\right|_{p}=a(p)$.

\section{Toric varieties and equivariant cohomology}

For a good reference on toric varieties, see [Fulton 1993]. Let $X$ be a smooth complete toric variety of dimension $n$. We denote the fan of $X$ by $\Sigma_{X}$, the lattice of $X$ by $N_{X}$, and the big torus by $T_{X}$. Let $Y$ be a smooth complete toric variety such that

(1) $N_{X} \subset N_{Y}$ is a finite-index sublattice, and

(2) $\Sigma_{X}$ is a refinement of $\Sigma_{Y}$ obtained by adding finitely many one-dimensional rays.

There is an obvious map of fans $v: \Sigma_{X} \rightarrow \Sigma_{Y}$ which induces a smooth map $\mu: X \rightarrow Y$. We call a map induced by such a morphism of fans a toric morphism. It is easy to verify that $\mu: T_{X} \rightarrow T_{Y}$ is a covering map with covering group $N_{Y} / N_{X}$. Thus, we may regard $Y$ as a $T_{X}$-space. Our goal in this section is to obtain a convenient description of the equivariant pushforward $\mu_{*}: H_{T}^{*}(X) \rightarrow H_{T}^{*}(Y)$ in terms of the combinatorics of $\Sigma_{X}$ and $\Sigma_{Y}$. Here $T=T_{X}$. 
We first note that fixed points $F$ of $X$ are in $1-1$ correspondence with $n$ dimensional cones $C_{F} \subset \Sigma_{X}$. Furthermore, the infinitesimal weights of the $T$ action on $N_{F}$ correspond to linear forms in $\operatorname{Hom}\left(N_{X}, \mathbb{Z}\right)$ which are dual to the generators of $C_{F}$ in $N_{X}$. With this in mind, we have the following theorem: Let $\mathbb{C}\left[\Sigma_{X}\right]$ denote the ring of piecewise polynomial functions on the fan of $X$. Then:

\section{Theorem 4.1.}

$$
H_{T}^{*}(X) \cong \mathbb{C}\left[\Sigma_{X}\right] .
$$

Proof. The map $H_{T}^{*}(X) \rightarrow \mathbb{C}\left[\Sigma_{X}\right]$ is defined by $\omega \mapsto\left\{\left.\omega\right|_{F}\right\}_{F \in X^{T}}$. That the polynomial functions $\left.\omega\right|_{F}$ piece together into a well-defined piecewise polynomial function follows from the fact that $\omega$ is a globally defined cohomology class. To define the reverse arrow, it suffices to describe it for piecewise linear functions. If $f \in \mathbb{C}\left[\Sigma_{X}\right]$ is piecewise linear, then it is well-known in toric geometry that $f$ defines a $T$-Cartier $\operatorname{divisor} \operatorname{div}(f)$. Let $f \mapsto \operatorname{div}(f)^{\#}$, where $\operatorname{div}(f)^{\#}$ denotes the equivariant extension whose restriction to a fixed point $F$ is $\left.f\right|_{F}$.

Via the identification $H_{T}^{*}(X) \cong \mathbb{C}\left[\Sigma_{X}\right]$, we define $v_{*}: \mathbb{C}\left[\Sigma_{X}\right] \rightarrow \mathbb{C}\left[\Sigma_{Y}\right]$ to be the map that makes the diagram

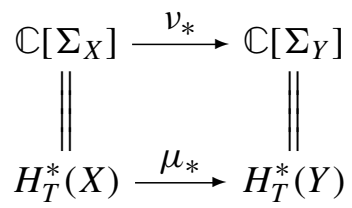

commute. Here we understand $\mathbb{C}\left[\Sigma_{Y}\right]$ to be the ring of piecewise polynomial functions on $\Sigma_{Y}$ with respect to the lattice $N_{X}$.

We now describe $v_{*}$ more explicitly. For $f \in \mathbb{C}\left[\Sigma_{X}\right], v_{*} f$ is given by viewing $\left.f\right|_{F}$ as the zero degree part of an equivariant cohomology class $\omega \in H_{T}^{*}(X)$, pushing $\omega$ forward by $\mu_{*}$, and then forming the piecewise polynomial function defined by the zero degree part of $\mu_{*} \omega$. Thus, let $C \subset \Sigma_{Y}$ be an $n$-dimensional cone. Let $v^{-1} C$ be the fan $\Sigma_{C} \subset \Sigma_{X}$ which is the union of $n$-dimensional cones $C_{i}$. Let $x_{1}^{C_{i}}, \ldots, x_{n}^{C_{i}}$ be the linear forms dual to $C_{i}$ and $x_{1}^{C}, \ldots, x_{n}^{C}$ the linear forms in $\operatorname{Hom}\left(N_{Y}, \mathbb{Z}\right) \subset \operatorname{Hom}\left(N_{X}, \mathbb{Z}\right)$ dual to $C$. By functorial localization we have

$$
\left(v_{*} f\right)_{C}=\sum_{C_{i} \subset \Sigma_{X}} f_{C_{i}} \frac{\prod_{j=1}^{n} x_{j}^{C}}{\prod_{j=1}^{n} x_{j}^{C_{i}}} .
$$

Similarly, we define $v^{*}: \mathbb{C}\left[\Sigma_{Y}\right] \rightarrow \mathbb{C}\left[\Sigma_{X}\right]$ as the map that makes the following diagram commute:

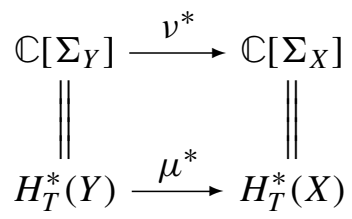




\section{Proposition 4.2.}

$$
v^{*}(f)=f \circ v .
$$

Proof. Let $\omega \in H_{T}^{*}(Y)$ be the form such that $\left.\omega\right|_{P}=\left.f\right|_{P}$ for every fixed point $P$. Let $F \in \mu^{-1}(P)$. Then

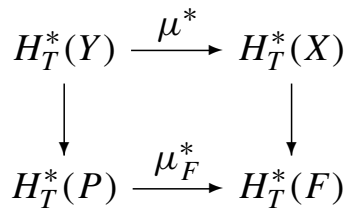

commutes. Hence $\left.\left(\mu^{*} \omega\right)\right|_{F}=\mu_{F}^{*}\left(\left.\omega\right|_{P}\right)=\mu_{F}^{*}\left(f_{P}\right)=f_{P}$. Thus $v^{*}(f)$ is the piecewise polynomial function which is equal to $f_{C_{P}}$ on every cone $C_{F} \in v^{-1} C_{P}$. This is precisely the piecewise polynomial $f \circ v$.

The map $v^{*}: \mathbb{C}\left[\Sigma_{Y}\right] \rightarrow \mathbb{C}\left[\Sigma_{X}\right]$ makes $\mathbb{C}\left[\Sigma_{X}\right]$ into a $\mathbb{C}\left[\Sigma_{Y}\right]$-module. This being so, we observe:

Proposition 4.3. $v_{*}$ is a $\mathbb{C}\left[\Sigma_{Y}\right]$-module homomorphism.

Proof. We wish to prove the projection formula $v_{*}\left(f v^{*} g\right)=v_{*}(f) \cdot g$. This follows from identifying $v_{*}$ with $\mu_{*}, v^{*}$ with $\mu^{*}$ and invoking the projection formula from equivariant cohomology.

\section{Push-forward formula for toroidal morphisms}

5.1. Definitions. Let $X$ be a compact complex manifold and $D_{X}=\sum_{I_{X}} D_{i}^{X}$ a divisor on $X$ whose irreducible components are smooth normal crossing divisors. For $I \subset I_{X}$, let $X_{I, j}$ denote the $j$-th connected component of $\bigcap_{I} D_{i}^{X}$. Let $X_{I, j}^{o}=$ $X_{I, j}-\bigcup_{I^{c}} D_{i}^{X}$. The collection of subvarieties $X_{I, j}^{o}$ form a stratification of $X$. Associated to these data is a polyhedral complex with integral structure defined as follows:

Corresponding to $X_{I, j}$, define $N_{I, j}=\mathbb{Z} e_{i_{1}, j}+\cdots+\mathbb{Z} e_{i_{k}, j}$ to be the free group on the elements $e_{i_{1}, j}, \ldots, e_{i_{k}, j}$. Here $i_{1}, \ldots i_{k}$ are the elements of $I$. Define $C_{I, j}$ to be the cone in the first orthant of this lattice. Whenever $I^{\prime} \subset I$ and $X_{I, j} \subset X_{I^{\prime}, j^{\prime}}$ we have natural inclusion maps $N_{I^{\prime}, j^{\prime}} \hookrightarrow N_{I, j}$ and $C_{I^{\prime}, j^{\prime}} \hookrightarrow C_{I, j}$. Define $\Sigma_{X}$ to be the polyhedral complex with integral structure obtained by gluing the cones $C_{I, j}$ together according to these inclusion maps.

Let $\mathbb{C}\left[\Sigma_{X}\right]$ denote the ring of piecewise polynomial functions on $\Sigma_{X}$. Fix $C \subset \Sigma_{X}$. Define $f^{C}$ to be the piecewise polynomial function which is equal to $\prod_{j=1}^{\operatorname{dim} C} x_{j}^{C}$ on every cone containing $C$, and equal to zero everywhere else. As in the toric geometry case, there is a natural correspondence between piecewise linear functions on $\Sigma_{X}$ and Cartier divisors whose irreducible components are components of $D_{X}$. We denote the piecewise linear function corresponding to $D$ by $f^{D}$. 
5.2. Toroidal morphisms. Our primary interest in this section is the study of toroidal morphisms, which are maps $\mu:\left(X, D_{X}, \Sigma_{X}\right) \rightarrow\left(Y, D_{Y}, \Sigma_{Y}\right)$ satisfying:

(1) $\mu: X-D_{X} \rightarrow Y-D_{Y}$ is an unramified cover.

(2) $\mu$ maps the closure of a stratum in $X$ to the closure of a stratum in $Y$.

(3) Let $U_{y}$ be an analytic neighborhood of $y \in Y$ such that the components of $D_{Y}$ passing through $y$ correspond to coordinate hyperplanes. Then for $x \in \mu^{-1}(y)$, there exists an analytic neighborhood $U_{x}$ of $x$ such that the components of $D_{X}$ passing through $x$ correspond to coordinate hyperplanes of $U_{x}$. Moreover, the map $U_{x} \rightarrow U_{y}$ is given by monomial functions in the coordinates.

Corresponding to $\mu$, we can define a map $v: \Sigma_{X} \rightarrow \Sigma_{Y}$ as follows: Let $C_{I, i} \subset \Sigma_{X}$ and let $e_{1}, \ldots, e_{k} \in N_{I, i}$ be the generators of $C_{I, i}$ which correspond to the divisors $D_{1}^{X}, \ldots, D_{k}^{X}$. We have that $\mu\left(X_{I, i}\right)=Y_{J, j}$. Let $v_{1}, \ldots, v_{\ell} \in N_{J, j}$ be the generators of $C_{J, j}$ which correspond to the divisors $D_{1}^{Y}, \ldots, D_{\ell}^{Y}$. For $1 \leq s \leq k, 1 \leq t \leq \ell$, define $a_{s t}$ to be the coefficient of $D_{s}^{X}$ of the divisor $\mu^{*}\left(D_{t}^{Y}\right)$. Then we define $v\left(e_{s}\right)=\sum a_{s t} v_{t}$. Note that if $\left(X, \Sigma_{X}\right) \rightarrow\left(Y, \Sigma_{Y}\right)$ is a smooth toric morphism of toric varieties, then $v: \Sigma_{X} \rightarrow \Sigma_{Y}$ is the natural morphism of polyhedral complexes.

Proposition 5.1. If $C=C_{J, j} \subset \Sigma_{Y}$, then $v^{-1} C$ is the union of fans $\Sigma_{\alpha} \subset \Sigma_{X}$ with the following properties:

(1) $\Sigma_{\alpha}$ is a refinement of $C$ obtained by adding finitely many one-dimensional rays.

(2) The lattice $N_{\alpha}$ of $\Sigma_{\alpha}$ is a finite-index sublattice of $N_{C}$.

(3) The fans $\Sigma_{\alpha}$ are in $1-1$ correspondence with connected components $U_{\alpha}$ of $\mu^{-1}\left(N_{Y_{J, j}^{o}}\right)$. The map $U_{\alpha} \rightarrow N_{Y_{J, j}^{o}}$ is a fibration given by the smooth toric morphism $\mathbf{P}_{\Sigma_{\alpha}, N_{\alpha}} \rightarrow \mathbf{P}_{C, N_{C}}$ along the fiber, and a $d_{\alpha}=d\left(\Sigma_{\alpha}\right)$-cover of $Y_{J, j}^{o}$ along the base.

For a proof, see [Borisov and Libgober 2005]. In the examples studied in this paper, it is easy to see that the proposition holds. For the purposes of this paper, therefore, one may take Proposition 5.1 as an axiom.

5.3. Pushforward formula for polyhedral complexes. Motivated by the description of the push-forward $v_{*}$ for toric morphisms, define $v_{*}: \mathbb{C}\left[\Sigma_{X}\right] \rightarrow \mathbb{C}\left[\Sigma_{Y}\right]$ as follows. Let $C \subset \Sigma_{Y}$ be an $n$-dimensional cone with dual linear forms $x_{1}^{C}, \ldots, x_{n}^{C}$. Then for $f \in \mathbb{C}\left[\Sigma_{X}\right]$, we define

$$
\left(v_{*} f\right)_{C}=\sum_{\alpha} d_{\alpha} \sum_{C_{i} \in \Sigma_{\alpha}} f_{C_{i}} \cdot \frac{\prod_{j=1}^{n} x_{j}^{C}}{\prod_{j=1}^{n} x_{j}^{C_{i}}} .
$$

The second sum is taken over the cones $C_{i} \subset \Sigma_{\alpha}$ with the same dimension as $C$. 
Let $V$ be the toric variety $\coprod_{\alpha} d_{\alpha} \cdot \mathbf{P}_{\Sigma_{\alpha}, N_{\alpha}}$ with polyhedral fan $\Sigma_{V}$. We have a natural toric morphism $V \rightarrow \mathbb{C}^{n}$. We can compactify $V$ and $\mathbb{C}^{n}$ to obtain a smooth toric morphism $\bar{V} \rightarrow \mathbb{P}^{n}$. If we view $f$ as a piecewise polynomial function on the fan of $\bar{V}$, then the above formula simply corresponds to $\left(v_{*} f\right)_{C}$ where $v: \Sigma_{\bar{V}} \rightarrow$ $\Sigma_{\mathbb{P} n}$. This identification allows us to apply the tools of the previous section toward the study of $v_{*}$.

We first observe that $\left(v_{*} f\right)_{C}$ is indeed a polynomial function. This follows from the above identification of $v_{*}$ with the equivariant pushforward of a toric morphism. Furthermore, if we define $v^{*}: \mathbb{C}\left[\Sigma_{Y}\right] \rightarrow \mathbb{C}\left[\Sigma_{X}\right]$ by the formula $v^{*}(f)=f \circ v$, then the projection formula

$$
v_{*}\left(f v^{*} g\right)_{C}=v_{*}(f)_{C} \cdot g_{C}
$$

follows from the projection formula in equivariant cohomology.

Proposition 5.2. $v_{*}(f)$ is a piecewise polynomial function.

Proof. We first show that $v_{*}\left(f^{C}\right)$ is piecewise polynomial.

Fix $f=f^{C}$. Suppose $v(C) \subset C_{0}$ for some $C_{0} \subset \Sigma_{Y}$ of dimension $k=\operatorname{dim} C$. Then $v_{*}(f)_{C_{0}}=d\left(\Sigma_{C_{0}}\right) \prod_{j=1}^{k} x_{j}^{C_{0}}$. Suppose $C_{1}$ is a cone containing $C_{0}$. We wish to show $\left(v_{*} f\right)_{C_{1}}$ is an extension of $\left(v_{*} f\right)_{C_{0}}$.

Consider the toric morphism $\sigma: \mathbf{P}_{\Sigma_{C_{1}}, N\left(\Sigma_{C_{1}}\right)} \rightarrow \mathbb{C}^{\operatorname{dim} C_{1}}$ induced by the map $v$ : $\Sigma_{C_{1}} \rightarrow C_{1}$. Let $D_{1}, \ldots, D_{k}$ be the divisors in $\mathbf{P}_{\Sigma_{C_{1}}, N\left(\Sigma_{C_{1}}\right)}$ which correspond to the generators of $C$. Then the piecewise polynomial function $f \in \mathbb{C}\left[\Sigma_{C_{1}}\right]$ represents the equivariant Thom class of $D_{1} \cap \cdots \cap D_{k}$. Since $\sigma\left(D_{1} \cap \cdots \cap D_{k}\right)$ is the affine subspace of $\mathbb{C}^{\operatorname{dim} C_{1}}$ corresponding to $C_{0}$, we have that $\sigma_{*}(f)$ is the degree of $\sigma$ along $D_{1} \cap \cdots \cap D_{k}$ times the polynomial function which represents the equivariant Thom class of this subspace. But this implies that

$$
v_{*}(f)_{C_{1}}=d\left(\Sigma_{C_{1}}\right) \frac{\left[N\left(\Sigma_{C_{1}}\right): N\left(C_{1}\right)\right]}{\left[N\left(\Sigma_{C_{0}}\right): N\left(C_{0}\right)\right]} \prod_{j=1}^{k} x_{j}^{C_{0}}=d\left(\Sigma_{C_{0}}\right) \prod_{j=1}^{k} x_{j}^{C_{0}} .
$$

We need to explain the last equality. If $C_{0}$ corresponds to the strata $Y_{I, j}^{o}$ and $U \rightarrow N_{Y_{I, j}^{o}}$ is the fibration in Proposition 5.1 corresponding to the subdivision $\Sigma_{C_{0}}$, then $d\left(\Sigma_{C_{0}}\right)\left[N\left(\Sigma_{C_{0}}\right): N\left(C_{0}\right)\right]$ and $d\left(\Sigma_{C_{1}}\right)\left[N\left(\Sigma_{C_{1}}\right): N\left(C_{1}\right)\right]$ both give the number of points in the preimage of a generic point in $N_{Y_{I, j}^{o}}$.

Next suppose that $C$ is mapped to a cone $C_{0}$ of strictly larger dimension. Consider the toric morphism $\mathbf{P}_{\Sigma_{C_{0}}, N\left(\Sigma_{C_{0}}\right)} \rightarrow \mathbf{P}_{C_{0}, N\left(C_{0}\right)}$ induced by the map $v: \Sigma_{C_{0}} \rightarrow$ $C_{0}$. The polynomial function $f \in \mathbb{C}\left[\Sigma_{C_{0}}\right]$ represents the Thom class of an exceptional toric subvariety. Thus $\nu_{*}(f)=0$, and it is easy to verify that $v_{*}(f)=0$ on every cone containing $C_{0}$. Thus, $\nu_{*}$ maps the elements $f^{C}$ to piecewise polynomial functions. Since these functions generate $\mathbb{C}\left[\Sigma_{X}\right]$ as a $\mathbb{C}\left[\Sigma_{Y}\right]$-module, the proposition follows from the projection formula. 
In what follows we assume that $\mu: X \rightarrow Y$ is an equivariant map of compact $T$ spaces. Furthermore, we assume that the irreducible components of $D_{X}$ and $D_{Y}$ are invariant under the $T$-action. Define a map $\rho_{X}: \mathbb{C}\left[\Sigma_{X}\right] \rightarrow H_{T}^{*}(X)$ as follows: Fix a cone $C=C_{I, i}$ which corresponds to a connected component of the intersection locus of the divisors $D_{1}, \ldots, D_{k}$. Define

$$
\rho_{X}\left[f^{C} \cdot\left(f^{D_{1}}\right)^{a_{1}} \ldots\left(f^{D_{k}}\right)^{a_{k}}\right]=\Phi_{X_{I, i}} \wedge D_{1}^{a_{1}} \wedge \cdots \wedge D_{k}^{a_{k}} .
$$

Here $\Phi_{X_{I, i}}$ denotes the (extension by zero) equivariant Thom class of $X_{I, i} \subset X$ and, by abuse of notation, $D_{j}$ denote the (extensions by zero) equivariant Thom classes of the divisors $D_{j}$.

Lemma 5.3. $\rho_{X}$ is a ring homomorphism.

Proof. Fix cones $C_{1}=C_{I_{1}, i_{1}}$ and $C_{2}=C_{I_{2}, i_{2}}$. It suffices to prove the theorem for the polynomials $f^{C_{1}}$ and $f^{C_{2}}$. Let $I=I_{1} \cup I_{2}$. Let $C_{I, i}$ denote the cones which correspond to components of the intersection $X_{I_{1}, i_{1}} \cap X_{I_{2}, i_{2}}$. Clearly

$$
f^{C_{1}} f^{C_{2}}=\sum_{I, i} f^{C_{I, i}} \prod_{I_{1} \cap I_{2}} f^{D_{j}}
$$

Thus $\rho_{X}\left(f^{C_{1}} f^{C_{2}}\right)=\sum_{I, i} \Phi_{X_{I, i}} \prod_{I_{1} \cap I_{2}} D_{j}$. But by the equivariant version of the excess intersection formula, this is precisely the formula for $\rho_{X}\left(f^{C_{1}}\right) \rho_{X}\left(f^{C_{2}}\right)$.

\section{Lemma 5.4.}

$$
\rho_{X} v^{*}=\mu^{*} \rho_{Y} .
$$

Proof. It suffices to check this for polynomials $f^{C_{I, k}}$. If $D$ is a divisor on $Y$ whose irreducible components are components of $D_{Y}$, then $v^{*} f^{D}$ is the piecewise linear function corresponding to $\mu^{*} D$. It follows that $\rho_{X} \nu^{*} f^{D}=\mu^{*} \rho_{Y} f^{D}$. Since all the maps are ring homomorphisms, this implies that $\rho_{X} v^{*} \prod_{j \in I} f^{D_{j}}=$ $\mu^{*} \rho_{Y} \prod_{j \in I} f^{D_{j}}$. Let $\mu^{*} D_{i}=\sum_{j} a_{i j} E_{j}$ as Cartier divisors. As in the lemma in the Appendix, choose equivariant Thom forms $\Phi_{E_{j}}$ and $\Phi_{D_{i}}$ with support in small tubular neighborhoods of their respective divisors so that

$$
\mu^{*} \Phi_{D_{i}}=\sum_{j} a_{i j} \Phi_{E_{j}}+d \psi_{i}
$$

as forms. Here $\psi_{i}$ are equivariant forms with compact support in $\mu^{-1} N_{D_{i}}$. Let $\{I, k\}$ index the connected components of $\bigcap_{I} D_{i}$. If we choose $N_{D_{i}}$ sufficiently small, then

$$
\prod_{I} \Phi_{D_{i}}=\sum_{I, k}\left(\prod_{I} \Phi_{D_{i}}\right)_{I, k}
$$

where $\left(\prod_{I} \Phi_{D_{i}}\right)_{I, k}$ is the extension by zero of the form $\left.\prod_{I} \Phi_{D_{i}}\right|_{N_{I, k}}$. 
Now $\prod_{I} f^{D_{i}}=\sum f^{C_{I, k}}$ and clearly $\left(\prod_{I} \Phi_{D_{i}}\right)_{I, k}$ is a representative of $\rho_{Y}\left(f^{C_{I, k}}\right)$. We have that

$$
\mu^{*}\left(\prod_{I} \Phi_{D_{i}}\right)_{I, k}=\left(\prod_{I}\left(\sum_{j} a_{i j} \Phi_{E_{j}}+d \psi_{i}\right)\right)_{\mu^{-1} N_{I, k}},
$$

where the subscript $\mu^{-1} N_{I, k}$ means the extension by zero of the form restricted to this open set. Since the $\psi_{i}$ forms have compact support in $\mu^{-1} N_{D_{i}}$, this form is cohomologous to

$$
\left(\prod_{I} \sum_{j} a_{i j} \Phi_{E_{j}}\right)_{\mu^{-1} N_{I, k}}
$$

But this is in turn a representative of $\rho_{X} v^{*} f^{C_{I, k}}$.

Lemma 5.5. $\mu_{*} \rho_{X}=\rho_{Y} v_{*}$.

Proof. Since $\rho_{X} v^{*}=\mu^{*} \rho_{Y}$ and the polynomials $f^{C}$ generate $\mathbb{C}\left[\Sigma_{X}\right]$ as a $\mathbb{C}\left[\Sigma_{Y}\right]$ module, by the projection formula it suffices to check $\mu_{*} \rho_{X} f^{C}=\rho_{Y} v_{*} f^{C}$.

Case 1: $C_{I, i}$ is mapped by $v$ to a cone $C_{J, j}$ of the same dimension. From the proof of Proposition 5.2, $v_{*} f^{C_{I, i}}=d f^{C_{J, j}}$ where $d$ is the degree of $\mu: X_{I, i} \rightarrow Y_{J, j}$. Thus, $\rho_{Y} v_{*} f^{C_{I, i}}=d \Phi_{Y_{J, j}}=\mu_{*} v_{*} f^{C_{I, i}}$.

Case 2: $C_{I, i}$ is mapped by $v$ into a cone of strictly larger dimension. As shown in Proposition 5.2, $v_{*} f^{C_{I, i}}=0$, so $\rho_{Y} v_{*} f^{C_{I, i}}=0=\mu_{*} \Phi_{X_{I, i}}=\mu_{*} \rho_{X} f^{C_{I, i}}$.

Remark 5.6. It is clear that these lemmas relating $\mu$ to $v$ extend without difficulty to the ring $\mathbb{C} \llbracket \Sigma_{X} \rrbracket$ of piecewise convergent power series.

\section{A rigidity theorem for elliptic genera on toric varieties}

For $X$ a toric variety and $D \subset X$ a $T$-Cartier divisor, the equivariant elliptic genus of the pair $(X, D)$ may be interpreted as the equivariant index of an associated differential operator. In this section we prove that the equivariant index of this operator is actually zero whenever $(X, D)$ satisfies the Calabi-Yau condition $K_{X}+$ $D=0$. This rigidity result closely resembles results by Hattori on the elliptic genera of multifans [Hattori 2006]. As we will see, this rigidity theorem is actually closely related to the change of variable formula for the elliptic genus. We first define the operator and prove its rigidity.

Let $X$ be a smooth complete toric variety of dimension $n$. Let $T=\left(S^{1}\right)^{n}$. We can think of $T$ as sitting inside the big-torus of $X$; as such, it induces a natural action on $X$ with isolated fixed points. Let $D_{1}, \ldots, D_{\ell}$ be the $T$-invariant divisors corresponding to the one-dimensional cones on the fan of $X$. Suppose $K_{X}+\sum_{i} \delta_{i} D_{i}=0$ for integers $\delta_{i} \neq 1$. Call such a pair $\left(X, \sum_{i} \delta_{i} D_{i}\right)$ a toric Calabi-Yau pair. Define 
$\mathscr{E} \mathscr{L} \mathscr{L}\left(\sum_{i} \delta_{i} D_{i}\right)$ to be the following vector bundle over $X$ :

$\bigotimes_{i}\left(\theta^{\prime}(0) \bigotimes_{n=1}^{\infty} \Lambda_{-y^{-\delta_{i}+1} q^{n-1}} \mathcal{O}\left(-D_{i}\right) \otimes \Lambda_{-y^{\delta_{i}-1} q^{n}} \mathcal{O}\left(D_{i}\right) \otimes S_{q^{n}} \mathscr{O}\left(-D_{i}\right) \otimes S_{q^{n}} \mathscr{O}\left(D_{i}\right)\right)$.

The modular properties of the ordinary index of this operator were discussed by Borisov and Gunnells [2001]. However, they did not prove the rigidity of the equivariant index.

Theorem 6.1. The equivariant index of $\mathscr{E} \mathscr{L} \mathscr{L}$ is identically zero.

Proof. We use a modularity argument similar to the one in [Liu 1996]. It suffices to prove that $\mathscr{E} \mathscr{L} \mathscr{L}$ is rigid under the action of a generic 1-parameter subgroup $S^{1} \subset T$. We may further assume that this $S^{1}$ action has isolated fixed points. If $p$ is a fixed point of this action, we must have $p=D_{i_{1}} \cap \cdots \cap D_{i_{n}}$ for some choice of indices $i_{k}$ depending on $p$. Take $I_{p}=\left\{D_{i_{1}}, \ldots, D_{i_{n}}\right\}$ and let $I_{p}^{c}$ consist of the remaining $T$-invariant divisors on $X . T_{p} X$ splits as $T_{p} X=\left.\mathcal{O}\left(D_{i_{1}}\right) \oplus \cdots \oplus \mathcal{O}\left(D_{i_{n}}\right)\right|_{p}$.

Thus, if the exponents of the $S^{1}$ action on $\mathcal{O}\left(D_{i}\right)$ are $m_{i}$, then the exponents of the action on $T_{p} X$ are $m_{i_{1}}, \ldots, m_{i_{n}}$. By the fixed point formula, the equivariant index of $\mathscr{E} \mathscr{L} \mathscr{L}$ is given, up to a normalization factor which is independent of $t$ and the fixed points $\{p\}$, by

$$
\sum_{p} \prod_{I_{p}} \frac{\theta\left(m_{i} t-\left(-\delta_{i}+1\right) z, \tau\right)}{\theta\left(m_{i} t, \tau\right)} \prod_{I_{p}^{c}} \theta\left(-\left(-\delta_{j}+1\right) z, \tau\right) .
$$

Call this function $F(t, z, \tau)$. Here $\tau \in \mathbb{H}$ is the lattice parameter defining the Jacobi theta function $\theta(t, \tau)$.

Since $F(t, z, \tau)$, for $t \in \mathbb{R}$, is the index of an elliptic operator, $F(t, z, \tau)$ is holomorphic for $(t, z, \tau) \in \mathbb{R} \times \mathbb{C} \times \mathbb{M}$. We first examine the modular properties of $F$. Define an action of $\operatorname{SL}(2, \mathbb{Z})$ on $\mathbb{C} \times \mathbb{C} \times \mathbb{M}$ by

$$
\left(\begin{array}{ll}
a & b \\
c & d
\end{array}\right) \cdot(t, z, \tau)=\left(\frac{t}{c \tau+d}, \frac{z}{c \tau+d}, \frac{a \tau+b}{c \tau+d}\right) .
$$

If $g \in \operatorname{SL}(2, \mathbb{Z})$ and $F$ is a function on $\mathbb{C} \times \mathbb{C} \times \mathbb{H}$, we define $(g \cdot F)(t, z, \tau)=$ $F\left(g^{-1}(t, z, \tau)\right)$. Let $F$ be the function given by the fixed point formula above. From the relations

$$
\theta\left(\frac{t}{c \tau+d}, \frac{a \tau+b}{c \tau+d}\right)=\zeta(c \tau+d)^{1 / 2} e^{\pi i c t^{2} /(c \tau+d)} \theta(t, \tau)
$$

we conclude that

$$
F\left(\frac{t}{c \tau+d}, \frac{z}{c \tau+d}, \frac{a \tau+b}{c \tau+d}\right)
$$


is equal to

$$
\begin{aligned}
\sum_{p}\left(\prod_{I_{p}} \exp \frac{\pi i c\left(m_{i} t-\left(-\delta_{i}+1\right) z\right)^{2}}{c \tau+d} \cdot \exp \frac{c \tau+d}{\pi i c\left(m_{i} t\right)^{2}} \cdot \frac{\theta\left(m_{i} t-\left(-\delta_{i}+1\right) z, \tau\right)}{\theta\left(m_{i} t, \tau\right)}\right. \\
\left.\times \prod_{I_{p}^{c}} \exp \frac{\pi i c\left(\left(-\delta_{j}+1\right) z\right)^{2}}{c \tau+d} \zeta(c \tau+d)^{1 / 2} \theta\left(-\left(-\delta_{j}+1\right) z, \tau\right)\right) .
\end{aligned}
$$

This expression simplifies to

$$
\begin{aligned}
& \zeta^{\ell-n}(c \tau+d)^{(\ell-n) / 2} \exp \frac{\pi i c \sum_{i=1}^{\ell}\left(-\delta_{i}+1\right)^{2} z^{2}}{c \tau+d} \\
& \sum_{p}\left(\exp \frac{-2 \pi i c \sum_{I_{p}} m_{i}\left(-\delta_{i}+1\right) z t}{c \tau+d}\right. \\
&\left.\quad \times \prod_{I_{p}} \frac{\theta\left(m_{i} t-\left(-\delta_{i}+1\right) z, \tau\right)}{\theta\left(m_{i} t, \tau\right)} \prod_{I_{p}^{c}} \theta\left(-\left(-\delta_{j}+1\right) z, \tau\right)\right) .
\end{aligned}
$$

Since $K_{X}+\sum_{i} \delta_{i} D_{i}=0$ and $K_{X}=-\sum_{i} D_{i}$, we have $\sum_{i}\left(-\delta_{i}+1\right) D_{i}=0$. Thus, the weights at every fixed point for this trivial line bundle must be the same. But the weight at a fixed point $p$ is given by $\sum_{I_{p}}\left(-\delta_{i}+1\right) m_{i}$. Since this sum is independent of $p$, we can pull the terms

$$
\exp \frac{-2 \pi i c \sum_{I_{p}}\left(-\delta_{i}+1\right) m_{i} t z}{c \tau+d}
$$

outside of the summation over the fixed points. We therefore have $F(g(t, z, \tau))=$ $K_{g}(t, z, \tau) F(t, z, \tau)$ for some holomorphic nowhere zero function $K_{g}(t, z, \tau)$. In particular, $F(g(t, z, \tau))$ has no poles for $(t, z, \tau) \in \mathbb{R} \times \mathbb{C} \times \mathbb{U}$.

We now show that $F$ is in fact holomorphic for $(t, z, \tau) \in \mathbb{C} \times \mathbb{C} \times \mathbb{H}$. Clearly, the only poles for $F$ are of the form $\left(\frac{n}{\ell}\left(c \tau_{0}+d\right), z_{0}, \tau_{0}\right)$, where we can assume that $(c, d)=1$. Choose integers $a$ and $b$ so that $a d-b c=1$. Let $g=\left(\begin{array}{ll}a & c \\ b & d\end{array}\right)$. From the above, we know that $F$ is holomorphic at the point

$$
\left(\frac{n}{\ell}, \frac{z_{0}}{c \tau_{0}+d}, \frac{a \tau_{0}+b}{c \tau_{0}+d}\right)=g \cdot\left(\frac{n}{\ell}\left(c \tau_{0}+d\right), z_{0}, \tau_{0}\right) .
$$

This implies that $g^{-1} F$ is holomorphic at $\left(\frac{n}{\ell}\left(c \tau_{0}+d\right), z_{0}, \tau_{0}\right)$. But $\left(g^{-1} F\right)(t, z, \tau)$ is equal to $K_{g^{-1}}(t, z, \tau) F(t, z, \tau)$. Since $K_{g^{-1}}$ is holomorphic and nowhere vanishing, we must have that $F$ is holomorphic at $\left(\frac{n}{\ell}\left(c \tau_{0}+d\right), z_{0}, \tau_{0}\right)$. Therefore, $F$ is in fact holomorphic on $\mathbb{C} \times \mathbb{C} \times \mathbb{H}$.

Next we prove that $F(t, z, \tau)$ is constant in the variable $t$. Let $z=\frac{1}{N}$ for $N$ an integer. It is easy to verify that $F\left(t+1, \frac{1}{N}, \tau\right)=F\left(t, \frac{1}{N}, \tau\right)$ and $F\left(t+N \tau, \frac{1}{N}, \tau\right)=$ 
$F\left(t, \frac{1}{N}, \tau\right)$. Thus, $F\left(t, \frac{1}{N}, \tau\right)$ is a holomorphic function on a torus, and therefore constant. Hence, for every $N$,

$$
\frac{\partial}{\partial t} F\left(t, \frac{1}{N}, \tau\right)=0
$$

Hence, we must have $\frac{\partial}{\partial t} F(t, z, \tau)=0$. In other words, $F$ is constant in $t$.

Finally, since the coefficients $\delta_{i} \neq 1$, for a generic $S^{1}$ action the summation $\sum_{I_{p}}\left(-\delta_{i}+1\right) m_{i} \neq 0$. As in Hattori's proof [2006] of vanishing theorems for the elliptic genus of multifans, we get $F(t+\tau, z, \tau)=e^{2 \pi i \sum\left(-\delta_{i}+1\right) m_{i} z} F(t, z, \tau)=$ $F(t, z, \tau)$, which implies that $F \equiv 0$.

If $\left\{D_{i}\right\}_{i=1}^{\ell}$ are the $T$-Cartier divisors on a toric variety $X$, then $T X$ is stably equivalent to $\bigoplus_{i=1}^{\ell} O\left(D_{i}\right)$. By the Atiyah-Bott-Lefschetz fixed point formula, this implies that the equivariant index of $\mathscr{E} \mathscr{L} \mathscr{L}\left(\sum \delta_{i} D_{i}\right)$ corresponds to the equivariant elliptic genus $\int_{X} E l l_{T}\left(\sum \delta_{i} D_{i}\right)$, up to a normalization factor.

With this in mind, we turn our attention to the blow-up of $\mathbb{C}^{n}$ at the origin. Let $T=\left(S^{1}\right)^{n}$ act on $\mathbb{C}^{n}$ as $\left(t_{1} x_{1}, \ldots, t_{n} x_{n}\right)$. This induces a natural action on $\widetilde{\mathbb{C}^{n}}$. The fixed points of $\widetilde{\mathbb{C}^{n}}$ are the points $p_{i}=[0: \cdots: 1: \cdots: 0]$ in the exceptional divisor which have 1 in the $i$-th homogeneous coordinate and zero everywhere else. Set $t_{i}=e^{2 \pi i u_{i}}$. Then the infinitesimal weights at $p_{i}$ are $u_{1}-u_{i}, \ldots, u_{n}-u_{i}, u_{i}$.

For $i=1, \ldots, n$, let $\alpha_{i}<1$ be the coefficients of the coordinate hyperplanes $D_{i}$. Let $\alpha_{0}=\sum_{i=1}^{n} \alpha_{i}+(1-n)$. For this simple blow-up, the change of variable formula for the equivariant elliptic genus of $\left(\mathbb{C}^{n}, \sum \alpha_{i} D_{i}\right)$ takes on the form

\section{Lemma 6.2.}

$$
\begin{aligned}
& \sum_{i=1}^{n} \prod_{j \neq i}^{n} \frac{\theta\left(u_{j}-u_{i}-\left(-\alpha_{j}+1\right) z\right)}{\theta\left(u_{j}-u_{i}\right) \theta\left(-\left(-\alpha_{j}+1\right) z\right)} \cdot \frac{\theta\left(u_{i}-\left(-\alpha_{0}+1\right) z\right)}{\theta\left(u_{i}\right) \theta\left(-\left(-\alpha_{0}+1\right) z\right)} \\
&=\prod_{j=1}^{n} \frac{\theta\left(u_{j}-\left(-\alpha_{j}+1\right) z\right)}{\theta\left(u_{j}\right) \theta\left(-\left(-\alpha_{j}+1\right) z\right)} .
\end{aligned}
$$

More generally, let $\mu: X \rightarrow Y$ be a composition of toric blow-ups of a smooth complete toric variety $Y$ with associated simplicial map $v: \Sigma_{X} \rightarrow \Sigma_{Y}$. Since the map has degree $1, X$ and $Y$ share the same lattice $N$. For $i=1, \ldots, k$, let $a_{i}$ denote the 1-dimensional rays of $\Sigma_{X}$ and for $j=1, \ldots, \ell$, let $b_{j}$ denote the 1-dimensional rays of $\Sigma_{Y}$. Any sequence $\alpha=\left\{\alpha_{1}, \ldots, \alpha_{k}\right\}$ of rationals $\alpha_{i}<0$ defines a piecewise linear function $f_{\alpha} \in \mathbb{C}\left[\Sigma_{X}\right]$ given by $f_{\alpha}\left(a_{i}\right)=\alpha_{i}$. This linear function in turn gives rise to the $T$-Cartier divisor $\alpha_{1} D_{a_{1}}+\cdots+\alpha_{k} D_{a_{k}}$, where $D_{a_{i}}$ are the divisors associated to the rays $a_{i}$.

Clearly $\mu^{*}\left(\alpha_{1} D_{a_{1}}+\cdots+\alpha_{k} D_{a_{k}}\right)$ is the $T$-Cartier divisor on $Y$ corresponding to the linear function $v^{*} f_{\alpha}$. For each ray $b_{j}$, let $\beta_{j}=f_{\alpha}\left(b_{j}\right)$. Then the sequence 
$\beta=\left\{\beta_{1}, \ldots, \beta_{\ell}\right\}$ defines the piecewise linear function $v^{*} f_{\alpha}$ and corresponds to the divisor $\mu^{*}\left(\alpha_{1} D_{a_{1}}+\cdots+\alpha_{k} D_{a_{k}}\right)$. We call the sequence $\beta=\mu^{*} \alpha$ the pull-back of $\alpha$ by $\mu$.

For each cone $n$-dimensional cone $C_{i} \subset \Sigma_{X}$, let $x_{i j}$ denote the linear forms in $\operatorname{Hom}(N, \mathbb{Z})$ dual to the generators of $C_{i}$. Let $\beta_{i j} \in \beta$ denote the coefficients corresponding to the generators of $C_{i}$. For an $n$-dimensional cone $C_{i}^{\prime} \subset \Sigma_{Y}$, define $y_{i j}$ and $\alpha_{i j}$ similarly. Then:

\section{Theorem 6.3.}

$$
\sum_{C_{i} \subset \Sigma_{X}} \prod_{j=1}^{n} \frac{\theta\left(x_{i j}+\beta_{i j} z\right)}{\theta\left(x_{i j}\right) \theta\left(\beta_{i j} z\right)}=\sum_{C_{i}^{\prime} \subset \Sigma_{Y}} \prod_{j=1}^{n} \frac{\theta\left(y_{i j}+\alpha_{i j} z\right)}{\theta\left(y_{i j}\right) \theta\left(\alpha_{i j} z\right)} .
$$

Proof. By the naturality property of the integer sequences $\alpha$ and $\beta$, if the formula holds for a single blow-up, then it will hold for a composition of blow-ups. Therefore, we can restrict our attention to the case of a single subdivision. Now the divisor $\alpha_{1} D_{1}+\cdots+\alpha_{k} D_{k}=K_{Y}+\sum_{i=1}^{k}\left(\alpha_{i}+1\right) D_{a_{i}}$. Thus, the right-hand side of the equation in the theorem is just (up to a normalization factor) the equivariant elliptic genus of the pair $\left(Y, \sum_{i}\left(\alpha_{i}+1\right) D_{a_{i}}\right)$. Since $\mu^{*}\left(\alpha_{1} D_{1}+\cdots+\alpha_{k} D_{k}\right)=$ $\beta_{1} D_{b_{1}}+\cdots+\beta_{\ell} D_{b_{\ell}}=K_{X}+\sum_{j}\left(\beta_{j}+1\right) D_{b_{j}}$, the left hand side of the equation is the elliptic genus of $(X, D)$ where $K_{X}+D=\mu^{*}\left(K_{Y}+\sum_{i}\left(\alpha_{i}+1\right) D_{a_{i}}\right)$.

It clearly suffices to prove that for $C_{i}^{\prime} \subset \Sigma_{Y}$ an $n$-dimensional cone, the contributions to the RHS coming from $C_{i}^{\prime}$ correspond to the contributions to the LHS coming from the cones $C_{i} \subset \Sigma_{X}$ mapping into $C_{i}^{\prime}$. Given the above identifications, this amounts to proving the change of variable formula for the blow-up of $\mathbb{C}^{n}$ along a $T$-invariant subspace, with the standard torus action. Since every such blow-up may be viewed as a product of the identity map along $\mathbb{C}^{k}$ times the blow-up at the origin of $\mathbb{C}^{n-k}$, it suffices to prove Lemma 6.2

Compactify $\mathbb{C}^{n}$ be viewing it as a subset of $\mathbb{P}^{n}$, and extend the torus action in the obvious manner. We may similarly view $\widetilde{\mathbb{C}^{n}}$ as an open subset of the blowup of $\mathbb{P}^{n}$ at the origin. Both compactifications are toric varieties and the induced actions are consistent with the action of the big torus. Let $H \subset \mathbb{P}^{n}$ denote the hyperplane at infinity-that is, the hyperplane disjoint from the blow-up point $p_{0}=$ $[0: \cdots: 0: 1]$. Since all the divisors $D_{i}$ corresponding to coordinate hyperplanes passing through $p_{0}$ are linearly equivalent to $H$ and $K_{\mathbb{P}^{n}}=-(n+1) H$, the line bundle $L=K_{\mathbb{P} n}+\sum_{i=1}^{n} \alpha_{i} D_{i}+\left((n+1)-\sum_{i} \alpha_{i}\right) H$ is trivial. Thus, the equivariant elliptic genus $\int_{\mathbb{P}^{n}} \mathscr{E} l l_{T}\left(\mathbb{P}^{n}, L-K_{\mathbb{P} n}\right)$ is zero. Similarly, $f^{*} L=K_{\mathbb{P}^{n}}+\sum_{i=1}^{n} \alpha_{i} \widetilde{D}_{i}+$ $\alpha_{0} E+\left((n+1)-\sum_{i} \alpha_{i}\right) f^{*} H=0$, which implies that $\int_{\widetilde{\mathbb{P} n}} \mathscr{E} l_{T}\left(\widetilde{\mathbb{P} n}, f^{*} L-K_{\widetilde{\mathbb{P} n}}\right)=0$. Thus

$$
\int_{\widetilde{\mathbb{P} n}} \mathscr{E} l l_{T}\left(\widetilde{\mathbb{P}^{n}}, f^{*} L-K_{\widetilde{\mathbb{P} n}}\right)=\int_{\mathbb{P} n} \mathscr{E} l l_{T}\left(\mathbb{P}^{n}, L-K_{\mathbb{P} n}\right) .
$$


It is easy to see that the contribution to the left-hand integral coming from the fixed points mapping to $p_{0}$ is the LHS of the equation in Lemma 6.2. Similarly, the contribution to the right-hand integral which comes from the blow-up point $p_{0}$ is equal to the RHS of Lemma 6.2. Since $\mathbb{P}^{n}$ and $\widetilde{\mathbb{P}^{n}}$ are isomorphic away from these points, the contributions to the two integrals coming from the other fixed points are the same, and cancel from both sides of the equation. This proves Lemma 6.2 and completes the proof.

We will see shortly that the addition formula in Theorem 6.3 lies at the heart of the change of variable formula.

\section{Equivariant change of variables formula}

7.1. Preliminaries. Let $\left(X, D=\sum_{I_{X}} \alpha_{i} D_{i}, G\right)$ be a $G$-normal pair with $\alpha_{i}<1$ and with a $T$-action commuting with $G$ and acting invariantly on $D$. Assume that every component $X_{\gamma}^{g, h}$ of $X^{g, h}$ is a complete intersection of components of $D$. Let $f: \widetilde{X} \rightarrow X$ be the blow-up of $X$ along a smooth $G$-invariant subvariety whose components are complete intersections of components of $D$. Define $\widetilde{D}=\sum_{I_{\tilde{X}}} \delta_{j} \widetilde{D}_{j}$ so that $K_{\widetilde{X}}+\widetilde{D}=f^{*}\left(K_{X}+D\right)$. Note that since $\alpha_{i}<1$, the coefficient in front of $E$ is less than 1 . Our goal in this section is to prove an equivariant change of variable formula for the orbifold elliptic genus:

Theorem 7.1. With the above notation, fix a component $X_{\gamma}^{g, h}$ and let $\widetilde{X}_{\mu}^{g, h}$ be the components of $\widetilde{X}^{g, h}$ which map to $X_{\gamma}^{g, h}$. Then

$$
f_{*} \sum_{\mu} \mathscr{E} l l_{\mathrm{orb}}^{T}\left(\widetilde{D}, \widetilde{X}_{\mu}^{g, h}\right)=\mathscr{E} l l_{\mathrm{orb}}^{T}\left(D, X_{\gamma}^{g, h}\right) .
$$

Let $I_{X}$ index the irreducible components of $D$. Let $I_{\widetilde{X}}$ index the proper transforms of these components, plus the exceptional divisors. Let $\Sigma_{X}$ be the polyhedral complex associated to $\left\{D_{i}\right\}_{I_{X}}$ and let $\Sigma_{\widetilde{X}}$ be the polyhedral complex associated to $\left\{\widetilde{D}_{j}\right\}_{I_{\tilde{X}}}$. Note that if $X_{I_{k}, i_{k}}$ are the components of the blow-up locus, then $\Sigma_{\widetilde{X}}$ is obtained from $\Sigma_{X}$ by adding the ray through the point $(1, \ldots, 1)$ in each of the cones $C_{I_{k}, i_{k}}$. The map $\left(\tilde{X}, \Sigma_{\widetilde{X}}, \widetilde{D}\right) \rightarrow\left(X, \Sigma_{X}, D\right)$ clearly satisfies the axioms of a toroidal morphism. Before proceeding with the proof, we need to establish some cohomological properties of this toroidal morphism:

For any variety $X$ with normal crossing divisors $\left\{D_{i}\right\}_{I_{X}}$, let $\Omega(\log D)$ be the locally-free sheaf defined as follows: Let $U=\left\{\left(x_{1}, \ldots, x_{k}, x_{k+1}, \ldots, x_{n}\right)\right\}$ be a local coordinate system centered at $p$ whose coordinate hyperplanes $x_{k+1}=$ $0, \ldots, x_{n}=0$ correspond to the divisors $D_{k+1}, \ldots, D_{n} \in\left\{D_{i}\right\}$ passing through p. Then $\Omega(\log D)(U)$ is the $\mathcal{O}_{X}$-module generated by the forms $d x_{1}, \ldots, d x_{k}$, 
$d x_{k+1} / x_{k+1}, \ldots, d x_{n} / x_{n}$. We have the exact sequence of sheaves

$$
0 \rightarrow \Omega^{1} \rightarrow \Omega(\log D) \rightarrow \bigoplus \mathcal{O}_{D_{i}} \rightarrow 0 .
$$

The first map is the obvious inclusion. The second arrow is the residue map which takes a section $\omega=\sum_{i} f_{i} \frac{d x_{i}}{x_{i}}$ to $\left.\bigoplus_{i} f_{i}\right|_{D_{i}}$. It is clear that this map is zero precisely when $\omega$ defines a local holomorphic section of $T^{*} X$. From the exact sequence of sheaves

$$
0 \rightarrow \mathrm{O}\left(-D_{i}\right) \rightarrow \mathrm{O}_{X} \rightarrow \mathrm{O}_{D_{i}} \rightarrow 0
$$

we get that $\Omega(\log D)-T^{*} X=-\sum \mathcal{O}\left(-D_{i}\right)$ as stable vector bundles. Applying the dual of this formula to the varieties $(X, D)$ and $(\widetilde{X}, \widetilde{D})$ defined above and observing that $f^{*} \Omega(\log D)=\Omega(\log \widetilde{D})$, we arrive at the $K$-theoretic relation

$$
T \tilde{X}-f^{*} T X=\sum_{I_{\tilde{X}}} \mathscr{O}\left(\widetilde{D}_{j}\right)-\sum_{I_{X}} f^{*} \mathscr{O}\left(D_{i}\right) .
$$

The equality is on the level of stable equivalence. We claim that the equality holds in $K_{T}(\tilde{X})$. To prove this, we verify the equality at every fixed component $F \subset \tilde{X}$. Let $F \in f^{-1}(P)$ be a fixed component which maps to $P$. Denote by $i_{F}$ and $i_{P}$ the inclusions of $F$ and $P$ in $\widetilde{X}$ and $X$. Let $\widetilde{D}_{1}, \ldots, \widetilde{D}_{\ell}$ be the divisors on $\widetilde{X}$ which contain $F$, and $D_{1}, \ldots, D_{r}$ the divisors on $X$ containing $P$. Then $i_{P}^{*} T X=$ $T P \oplus N \oplus \bigoplus_{i=1}^{r} i_{P}^{*} \mathcal{O}\left(D_{i}\right)$. Here if $Z$ is the connected component of $\bigcap_{i=1}^{r} D_{i}$ containing $P$, then $N$ is the normal bundle of $P$ in $Z$. Similarly, $i_{F}^{*} T \tilde{X}=T F \oplus \widetilde{N} \oplus$ $\bigoplus_{j=1}^{\ell} i_{F}^{*} \mathcal{O}\left(\widetilde{D}_{j}\right)$, with $\tilde{N}$ defined similarly. A computation in coordinates reveals that $f^{*} N=\widetilde{N}$. Since $i_{F}^{*} f^{*}=f^{*} i_{P}^{*}$, we have

$$
i_{F}^{*} T \tilde{X}-i_{F}^{*} f^{*} T X=T F+\sum_{j=1}^{\ell} i_{F}^{*} \mathcal{O}\left(\widetilde{D}_{j}\right)-f^{*} T P-\sum_{i=1}^{r} i_{F}^{*} f^{*} \mathcal{O}\left(D_{i}\right) .
$$

However, by the nonequivariant formula for $T \widetilde{X}-f^{*} T X$ derived from the $\log$ complex, we have that

$$
T F-f^{*} T P=\sum_{E_{j} \cap F<F} i_{F}^{*} \mathcal{O}\left(\widetilde{D}_{j}\right)-\sum_{D_{i} \cap P<P} i_{F}^{*} f^{*} \mathcal{O}\left(D_{i}\right)
$$

where the sums are taken over the divisors which intersect properly with the fixed components. Since these bundles all carry trivial $T$-actions, the above formula holds in the equivariant category. Finally, observe that if $\widetilde{D}_{j}$ is disjoint from $F$ (resp. $D_{i}$ is disjoint from $P$ ) then $i_{F}^{*} O\left(\widetilde{D}_{j}\right)\left(\right.$ resp. $\left.i_{P}^{*} O\left(D_{i}\right)\right)$ is equivariantly trivial. Hence

$$
i_{F}^{*} T \tilde{X}-i_{F}^{*} f^{*} T X=\sum_{I_{\tilde{X}}} i_{F}^{*} \mathcal{O}\left(\widetilde{D}_{j}\right)-\sum_{I_{X}} i_{F}^{*} f^{*} \mathcal{O}\left(D_{i}\right) .
$$


From this we deduce an important formula relating the equivariant Chern roots of $\widetilde{X}$ to $X$ :

Lemma 7.2. Let $f: \widetilde{X} \rightarrow X,\left\{\widetilde{D}_{j}\right\}$, and $\left\{D_{i}\right\}$ be as above. Then

$$
\frac{c_{T}(T \tilde{X})}{f^{*} c_{T}(T X)}=\frac{\prod_{I_{\tilde{X}}}\left(1+c_{1}^{T}\left(\widetilde{D}_{j}\right)\right)}{\prod_{I_{X}}\left(1+f^{*} c_{1}^{T}\left(D_{i}\right)\right)} .
$$

7.2. Proof of the change of variables formula. The method of proof used here is adapted from Borisov and Libgober's calculation of the pushforward of the orbifold elliptic genus by a toroidal morphism [Borisov and Libgober 2005].

By Lemma 7.2, $\mathscr{E} l l_{\text {orb }}^{T}\left(\widetilde{D}, \widetilde{X}_{\mu}^{g, h}\right)$ is equal to

$$
\begin{aligned}
& \Phi_{\widetilde{X}_{\mu}^{g, h}}^{T} f^{*}(\left.\prod_{T X} \frac{\frac{x_{j}(t)}{2 \pi i} \theta\left(\frac{x_{j}(t)}{2 \pi i}-z\right) \theta^{\prime}(0)}{\theta\left(\frac{x_{j}(t)}{2 \pi i}\right) \theta(-z)} \prod_{I_{X}} \frac{\theta\left(\frac{D_{i}(t)}{2 \pi i}\right) \theta(-z)}{\frac{D_{i}(t)}{2 \pi i} \theta\left(\frac{D_{i}(t)}{2 \pi i}-z\right) \theta^{\prime}(0)}\right) \\
& \times \prod_{I_{\mu}^{g, h}} \frac{\theta\left(\frac{\widetilde{D}_{i}(t)}{2 \pi i}+\epsilon_{i}(g)-\epsilon_{i}(h) \tau-\left(-\delta_{i}+1\right) z\right) \theta^{\prime}(0)}{\theta\left(\frac{\widetilde{D}_{i}(t)}{2 \pi i}+\epsilon_{i}(g)-\epsilon_{i}(h) \tau\right) \theta\left(-\left(-\delta_{i}+1\right) z\right)} e^{2 \pi i\left(-\delta_{i}+1\right) \epsilon_{i}(h) z} \\
& \times \prod_{I_{\tilde{X}}-I_{\mu}^{g, h}} \frac{\frac{\widetilde{D}_{k}(t)}{2 \pi i} \theta\left(\frac{\widetilde{D}_{k}(t)}{2 \pi i}-\left(-\delta_{k}+1\right) z\right) \theta^{\prime}(0)}{\theta\left(\frac{\widetilde{D}_{k}(t)}{2 \pi i}\right) \theta\left(-\left(-\delta_{k}+1\right) z\right)}\left(\frac{2 \pi i \theta(-z)}{\theta^{\prime}(0)}\right)^{n} .
\end{aligned}
$$

Thus, in order to prove the change of variables formula, we are reduced to proving that

$$
\begin{gathered}
f_{*}\left(\sum_{\widetilde{X}_{\mu}^{g, h}} \prod_{I_{\mu}^{g, h}} \frac{\theta\left(\frac{\widetilde{D}_{i}(t)}{2 \pi i}+\epsilon_{i}(g)-\epsilon_{i}(h) \tau-\left(-\delta_{i}+1\right) z\right) \frac{\theta^{\prime}(0)}{2 \pi i}}{\theta\left(\frac{\widetilde{D}_{i}(t)}{2 \pi i}+\epsilon_{i}(g)-\epsilon_{i}(h) \tau\right) \theta\left(-\left(-\delta_{i}+1\right) z\right)} e^{2 \pi i\left(-\delta_{i}+1\right) \epsilon_{i}(h) z}\right. \\
\left.\times \prod_{I_{\tilde{X}}-I_{\mu}^{g, h}} \frac{\frac{\widetilde{D}_{i}(t)}{2 \pi i} \theta\left(\frac{\widetilde{D}_{i}(t)}{2 \pi i}-\left(-\delta_{i}+1\right) z\right) \theta^{\prime}(0)}{\theta\left(\frac{\widetilde{D}_{i}(t)}{2 \pi i}\right) \theta\left(-\left(-\delta_{i}+1\right) z\right)} \cdot \Phi_{\widetilde{X}_{\mu}^{g, h}}^{T}\right) \\
=\prod_{I_{\gamma}^{g, h}} \frac{\theta\left(\frac{D_{i}(t)}{2 \pi i}+\epsilon_{i}(g)-\epsilon_{i}(h) \tau-\left(-\alpha_{i}+1\right) z\right) \frac{\theta^{\prime}(0)}{2 \pi i}}{\theta\left(\frac{D_{i}(t)}{2 \pi i}+\epsilon_{i}(g)-\epsilon_{i}(h) \tau\right) \theta\left(-\left(-\alpha_{i}+1\right) z\right)} e^{2 \pi i\left(-\alpha_{i}+1\right) \epsilon_{i}(h) z} \\
\times \prod_{I_{X}-I_{\gamma}^{g, h}} \frac{\frac{D_{i}(t)}{2 \pi i} \theta\left(\frac{D_{i}(t)}{2 \pi i}-\left(-\alpha_{i}+1\right) z\right) \theta^{\prime}(0)}{\theta\left(\frac{D_{i}(t)}{2 \pi i}\right) \theta\left(-\left(-\alpha_{i}+1\right) z\right)} \cdot \Phi_{X_{\gamma}^{g, h} .}^{T} .
\end{gathered}
$$

Call the expression in the large parentheses $\Omega_{\gamma}^{g, h}$. For each $X_{\gamma}^{g, h}$, define a piecewise convergent power series $F_{\gamma}^{g, h} \in \mathbb{C} \llbracket \Sigma_{X} \rrbracket$ as follows: Let $C_{\gamma}^{g, h}$ be the cone which 
corresponds to $X_{\gamma}^{g, h}$. For $C=C_{I, j}$ a cone containing $C_{\gamma}^{g, h}$, define

$$
\left.F_{\gamma}^{g, h}\right|_{C}=\prod_{I} \frac{\frac{x_{i}^{C}}{2 \pi i} \theta\left(\frac{x_{i}^{C}}{2 \pi i}+\epsilon_{i}(g)-\epsilon_{i}(h) \tau-\left(-\alpha_{i}+1\right) z\right) \theta^{\prime}(0)}{\theta\left(\frac{x_{i}^{C}}{2 \pi i}+\epsilon_{i}(g)-\epsilon_{i}(h) \tau\right) \theta\left(-\left(-\alpha_{i}+1\right) z\right)} e^{2 \pi i\left(-\alpha_{i}+1\right) \epsilon_{i}(h) z} .
$$

Here for $i \in I, x_{i}^{C}$ are the linear functions dual to the generators of $C$. If $D_{i}$ are the divisors which correspond to the generators of $C$, then $\epsilon_{i}(g)$ and $\epsilon_{i}(h)$ are the infinitesimal weights of the $g$ and $h$ action on $\left.\mathcal{O}\left(D_{i}\right)\right|_{X_{\gamma}^{g, h}}$. Finally, $\alpha_{i}$ refer to the coefficients of these $D_{i}$ in the divisor $D$ defined in the statement of the theorem. Finally, for $C$ a cone not containing $C_{\gamma}^{g, h}$, we define $\left.F_{\gamma}^{g, h}\right|_{C}=0$. It is easy to see that $F_{\gamma}^{g, h}$ is a well-defined piecewise convergent power series and that

$$
\rho_{X}\left(F_{\gamma}^{g, h}\right)=\left(\frac{\theta^{\prime}(0)}{2 \pi i \theta(-z)}\right)^{n} \Omega_{\gamma}^{g, h}
$$

We define the piecewise convergent power series $\widetilde{F}_{\mu}^{g, h} \in \mathbb{C} \llbracket \Sigma_{\widetilde{X}} \rrbracket$ similarly. By Lemma 5.4, we have reduced the problem to proving that

$$
\nu_{*} \sum_{\mu} \widetilde{F}_{\mu}^{g, h}=F_{\gamma}^{g, h} .
$$

For each cone $C$ containing $C_{\gamma}^{g, h}, \Sigma_{C}=v^{-1} C$ is a subdivision of $C$ obtained by adding no more than one ray through the point $(1, \ldots, 1)$ in each subcone. It is clear that the cones $C_{\mu}^{g, h}$ must be cones inside the fan $\Sigma_{C}$. Moreover, every cone in $\Sigma_{C}$ with the same dimension as $C$ will contain exactly one $C_{\mu}^{g, h}$ as a subcone. (If it contained more than one, that would contradict the fact that the $\widetilde{X}_{\mu}^{g, h}$ s are disjoint.) Thus, to prove the formula, we may restrict all our attention to the morphisms $v: \Sigma_{C} \rightarrow C$ for $C \supset C_{\gamma}^{g, h}$. For $C_{j} \subset \Sigma_{C}\left(\operatorname{dim} C_{j}=\operatorname{dim} C\right)$, let $x_{i j}=x_{i}^{C_{j}}$ and similarly define $\epsilon_{i j}, \delta_{i j}$ in the obvious manner. Let $x_{i}=x_{i}^{C}$. By the pushforward formula for $v_{*}$, we are reduced to proving that

$$
\begin{gathered}
\sum_{C_{j} \subset \Sigma_{C}} \prod_{i} \frac{\theta\left(\frac{x_{i j}}{2 \pi i}+\epsilon_{i j}(g)-\epsilon_{i j}(h) \tau-\left(-\delta_{i j}+1\right) z\right) \frac{\theta^{\prime}(0)}{2 \pi i}}{\theta\left(\frac{x_{i j}}{2 \pi i}+\epsilon_{i j}(g)-\epsilon_{i j}(h) \tau\right) \theta\left(-\left(-\delta_{i j}+1\right) z\right)} e^{2 \pi i\left(-\delta_{i j}+1\right) \epsilon_{i j}(h) z} \\
=\prod_{i} \frac{\theta\left(\frac{x_{i}}{2 \pi i}+\epsilon_{i}(g)-\epsilon_{i}(h) \tau-\left(-\alpha_{i}+1\right) z\right) \frac{\theta^{\prime}(0)}{2 \pi i}}{\theta\left(\frac{x_{i}}{2 \pi i}+\epsilon_{i}(g)-\epsilon_{i}(h) \tau\right) \theta\left(-\left(-\alpha_{i}+1\right) z\right)} e^{2 \pi i\left(-\alpha_{i}+1\right) \epsilon_{i}(h) z} .
\end{gathered}
$$

To prove this, view $v: \Sigma_{C} \rightarrow C$ as a toric morphism. Let $N$ be the lattice corresponding to the toric varieties defined by the fans $\Sigma_{C}$ and $C$. The elements $g$ and $h$ act on $\mathbf{P}_{C, N}$ and $\mathbf{P}_{\Sigma_{C}, N}$ as elements of the big torus. As such, we may view $g$ and $h$ as elements of a sup-lattice $N^{\prime} \supset N$ of finite index. Under this identification, and 
using the transformation properties of the Jacobi theta function, we may assume $\epsilon_{i}(g)=x_{i}(g)$ and $\epsilon_{i j}(g)=x_{i j}(g)$.

Following the notation in the proof of Theorem 6.3, let $\alpha=\left\{\alpha_{i}-1\right\}$ and $\delta=$ $\left\{\delta_{j}-1\right\}$, where the indices range over all one-dimensional rays in $C$ and $\Sigma_{C}$. Clearly $\delta=v^{*} \alpha$. Let $f_{\alpha}$ be the linear function on $C$ induced by the multiindex $\alpha$. Then $\sum_{i}\left(\alpha_{i}-1\right) \epsilon_{i}(h)=f_{\alpha}(h)$. Similarly, $\sum_{j}\left(\delta_{i j}-1\right) \epsilon_{i j}(h)=f_{\delta}(h)=f_{v^{*} \alpha}(h)=$ $v^{*} f_{\alpha}(h)=f_{\alpha}(h)$. Thus, all the exponentials on both sides of the above equation are the same. Now the equation follows from Theorem 6.3 after substituting $x_{i}$ with $x_{i}+\epsilon_{i}(g)-\epsilon_{i}(h) \tau$. This completes the proof.

7.3. Localization change of variable formula. Suppose now that $f: \widetilde{X} \rightarrow X$ is a $T \times G$ invariant blow-up of open varieties with compact $T$-fixed components. Assume further that $f:(\widetilde{X}, \widetilde{D}) \rightarrow(X, D)$ admits a smooth equivariant compactification $f^{\prime}:\left(\widetilde{X}^{\prime}, \widetilde{D}^{\prime}\right) \rightarrow\left(X^{\prime}, D^{\prime}\right)$ so that the compactified pairs remain $G$-normal, and $f^{\prime}$ is again an equivariant blow-up. Let $P$ be a fixed component of $X$ and $\{F\}$ the collection of fixed components mapping to $P$. Then by the change of variable formula for the orbifold elliptic genus of the compactification, plus functorial localization, we get the following localized version of Theorem 7.1:

Corollary 7.3. $\quad \sum_{F, \mu} \int_{F} \frac{i_{F}^{*} \mathscr{E} l l_{\mathrm{orb}}^{T}\left(\widetilde{D}, \widetilde{X}_{\mu}^{g, h}\right)}{e(F)}=\int_{P} \frac{i_{P}^{* \mathscr{E}} l l_{\mathrm{orb}}^{T}\left(D, X_{\gamma}^{g, h}\right)}{e(P)}$.

\section{Equivariant indices of ALE spaces}

In this section we prove an equivariant elliptic genus analogue of the McKay correspondence for ALE spaces. Let $G \subset \mathrm{SU}(2)$ be a finite subgroup. Let $T=S^{1}$ act on $\mathbb{C}^{2}$ by the diagonal action. $T$ clearly commutes with $G$. Our first goal is to construct an equivariant resolution of singularities for $\mathbb{C}^{2} / G$.

Let $g, h \in G$ be commuting pairs. Assume one of $g$ or $h$ is nontrivial so that $\left(\mathbb{C}^{2}\right)^{g, h}=(0,0)$. Since $g$ and $h$ commute, they have a simultaneous eigenbasis $(a, b)$ and $(-b, a)$. Let $\ell=\mathbb{C}(a, b)$ and $\ell^{\perp}=\mathbb{C}(-b, a)$. Let $D_{\ell}=\{-b x+a y=0\}$ and $D_{\ell^{\perp}}=\{a x+b y=0\}$. Then $D_{\ell}, D_{\ell^{\perp}}$ are $(g, h) \times T$-invariant normal crossing divisors and $D_{\ell} \cap D_{\ell^{\perp}}=\left(\mathbb{C}^{2}\right)^{g, h}$.

Let $f: \widetilde{\mathbb{C}^{2}} \rightarrow \mathbb{C}^{2}$ be the blow-up of $\mathbb{C}^{2}$ at the origin with exceptional divisor $E$. Let $\widetilde{D_{\ell}}$ and $\widetilde{D_{\ell^{\perp}}}$ denote the proper transforms of $D_{\ell}$ and $D_{\ell^{\perp}}$. The collection of divisors $\left\{\widetilde{D}_{\ell}, \widetilde{D}_{\ell^{\perp}}\right\}$ over all commuting pairs $(g, h)$ plus the exceptional divisor $E$ form a system of $T$-invariant normal crossing divisors. Moreover, this system of divisors is $G$-invariant. This just follows from the fact that if $\ell$ is an eigenvector for $g$, then $h \cdot \ell$ is an eigenvector for $h g h^{-1}$. Let $X=\widetilde{\mathbb{C}^{2}}$, and label this system of divisors $\left\{D_{j}\right\}_{I_{X}}$. The following lemma demonstrates that $\left(X, \sum D_{j}, G\right)$ is, in the words of Batyrev [1999], a canonical abelianization of $\left(\mathbb{C}^{2}, 0, G\right)$ : 
Lemma 8.1. $\left(X, \sum_{j} D_{j}, G\right)$ is $G$-normal with abelian stabilizers.

Proof. We first prove $G$-normality. Suppose $g \cdot x=x$ and $x \in D_{j}$, for some $g \neq e$. Then $x=\ell \in E$, since $G$ acts freely on $X-E$. Identifying $\ell$ with a line in $\mathbb{C}^{2}$, we see that $\ell$ is an eigenspace for $g$, and therefore, that $D_{j}=\widetilde{D}_{\ell}$. It follows that $g \cdot D_{j}=D_{j}$, which proves $G$-normality.

Next, let $g, h$ be two elements which fix $\ell$. Then $\ell$ and $\ell^{\perp}$ are orthogonal eigenspaces for $g$ and $h$. It follows that $g$ and $h$ are simultaneously diagonalizable, and therefore commute. This completes the proof.

Note that the above proof also implies that $X^{g}=\bigcap_{J} D_{j}$ for some indexing set $J \subset I_{X}$.

Consider the map $f: X \rightarrow X / G$. Let $p \in X$ have a nontrivial stabilizer $G_{p}$. Notice that $p \in X^{T}$ and $p=D_{p} \cap E$ for some divisor $D_{p} \in\left\{D_{j}\right\}_{I_{X}}$. Let $U_{p}=$ $\{(x, y)\}$ be a coordinate system centered at $p$ such that $\{x=0\}=E \cap U_{p}$ and $\{y=0\}=D_{p} \cap U_{p}$. Then $f\left(U_{p}\right) \cong U_{p} / G_{p}$. Since $\left(X, \sum_{j} D_{j}, G\right)$ is $G$-normal, we may identify $G_{p}$ as a finite subgroup of $S^{1} \times S^{1}$ acting on $U_{p}$ in the obvious manner. Thus, let

$$
\widetilde{U_{p} / G_{p}}
$$

be a toric resolution of the singularity at the origin of $U_{p} / G_{p}$. Repeat this procedure for every singular point $f(p) \in X / G$. We get a resolution of singularities $Y \rightarrow X / G$. Since all the $p$ are fixed points of $T, Y \rightarrow X / G$ is $T$-equivariant. The regular map $Y \rightarrow X / G \rightarrow \mathbb{C}^{2} / G$ gives us our desired $T$-equivariant resolution $\pi: Y \rightarrow \mathbb{C}^{2} / G$. Let $\left\{D_{i}\right\}_{I_{Y}}$ denote the set of divisors on $Y$ containing the exceptional curves of $\pi$ plus the proper transforms of the divisors $f\left(D_{j}\right)$ for $j \in I_{X}$. Then $\left\{D_{i}\right\}_{I_{Y}}$ is a system of $T$-invariant normal crossing divisors on $Y$.

Since the techniques of Section 5.3 pertain to compact varieties, we now describe some natural compactifications of $X$ and $Y$. We may view $\mathbb{C}^{2}=\{(x, y)\}$ as sitting inside $\mathbb{P}^{2}=\{[x: y: z]\}$ with the actions of $G$ and $T$ extending to $\mathbb{P}^{2}$ in the obvious manner. Let $\bar{X} \supset X$ be the blow-up of $\mathbb{P}^{2}$ at the origin. Notice that $\bar{X} / G$ is smooth along the hyperplane at infinity. Thus, let $\bar{Y}$ be the resolution of $\bar{X} / G$ that is equal to $\bar{X} / G$ at infinity and coincides with $Y$ everywhere else. Let $\left\{\bar{D}_{j}\right\}_{I_{X}}$ and $\left\{\bar{D}_{i}\right\}_{I_{Y}}$ denote the closures of the corresponding divisors on $X$ and $Y$. Note that the compactified divisors $\left\{\bar{D}_{j}\right\}_{I_{X}}$ and $\left\{\bar{D}_{i}\right\}_{I_{Y}}$ do not contain any new points in their intersection loci. Finally let $\Sigma_{\bar{X}}$ and $\Sigma_{\bar{Y}}$ be the polyhedral complexes associated to the above $T$-invariant normal crossing divisors.

We now describe the relationship between the polyhedral complexes of $\bar{X}$ and $\bar{Y}$. We first set up some notation. As above, let $f: \bar{X} \rightarrow \bar{X} / G$ be the global quotient. Let $p \in \bar{D}_{p} \cap E$. Then $p$ corresponds to a 2-dimensional cone $C_{p} \subset \Sigma_{\bar{X}}$ with lattice $N_{p}$. Let $C_{g \cdot p}$ be the corresponding 2-dim cone for each point $g \cdot p$ in the 
$G$-orbit of $p$. We have an obvious identification $N_{g \cdot p} \cong N_{p}$. Let $\Sigma_{f(p)}$ be the fan that corresponds to the toric desingularization $(*)$. Let $N_{f(p)}$ denote the refinement of the lattice $N_{p}$ which induces the toric quotient map $\mathbb{C}^{2} \rightarrow \mathbb{C}^{2} / G_{p}$. Next, if $D$ is a divisor in $\left\{\bar{D}_{j}\right\}_{I_{X}}$, let $C_{D}$ denote the corresponding ray in $\Sigma_{\bar{X}}$, with lattice $N_{D} \cong \mathbb{Z}$. We define $N_{f(D)}$ to be the superlattice of $N_{D}$ whose index is equal to the ramification index of $f$ along $D$. Given these data, we obtain the lattice $\Sigma_{\bar{Y}}$ from $\Sigma_{\bar{X}}$ as follows: First identify all the cones $\left(C_{g \cdot p}, N_{p}\right)$ for every point $g \cdot p$ in the $G$-orbit of $p$. Next replace their representative with the toric fan $\left(\Sigma_{f(p)}, N_{f(p)}\right)$. Finally, replace the rays $\left(C_{D}, N_{D}\right)$ with the rays $\left(C_{f(D)}, N_{f(D)}\right)$.

Unfortunately, the map $\bar{X} \rightarrow \bar{Y}$ is not regular. To remedy this, for every 2dimensional cone $C_{p} \subset \Sigma_{\bar{X}}$, let $\Sigma_{p}$ be a subdivision of $\Sigma_{f(p)}$ such that the morphism $\left(\Sigma_{p}, N_{p}\right) \rightarrow\left(\Sigma_{f(p)}, N_{f(p)}\right)$ induces a smooth map of toric varieties. Modify $\Sigma_{\bar{X}}$ by replacing every cone $C_{p}$ with $\Sigma_{p}$. Naturally, we identify $\Sigma_{g \cdot p}=\Sigma_{p}$ for every $g \in G$. Define $\hat{X}$ to be the toroidal modification of $\bar{X}$ whose polyhedral complex $\Sigma_{\hat{X}}$ corresponds to the above described modification of $\Sigma_{\bar{X}}$. It is easy to see that $\hat{X} \rightarrow \bar{X}$ factors into a sequence of $G \times T$-equivariant blow-ups at complete intersection points. Moreover, the smooth $T$-map $\mu: \hat{X} \rightarrow \bar{Y}$ is a toroidal morphism. We have the commutative diagram

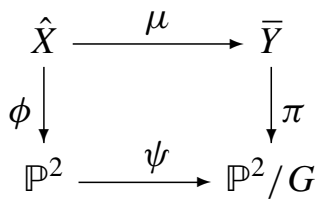

Let $\left\{\hat{D}_{j}\right\}_{j \in I_{\hat{X}}}$ denote the $T$-invariant normal crossing divisors on $\hat{X}$ which correspond to the 1 -dimensional rays of $\Sigma_{\hat{X}}$. We have that $\phi^{*} K_{\mathbb{P}^{2}}=K_{\hat{X}}+\sum_{I_{\hat{X}}} \beta_{j} \hat{D}_{j}$ for some integers $\beta_{j}<1$.

Lemma 8.2.

$$
\phi_{*} \mathscr{E} l l_{\text {orb }}^{T}\left(\hat{X}, \sum \beta_{j} \hat{D}_{j}, G\right)=\mathscr{E} l l_{\text {orb }}^{T}\left(\mathbb{P}^{2}, 0, G\right) .
$$

Proof. This follows almost directly from the change of variables formula for the orbifold elliptic genus discussed in the previous section. The only point which requires comment is the fact that the divisors $\left\{\phi\left(\hat{D}_{j}\right)\right\}_{I_{\hat{X}}-I_{\text {excep }}}$ (where $I_{\text {excep }}$ ranges over the exceptional curves) have their base locus at $[0: 0: 1]$ and therefore do not form a system of normal crossing divisors. However, for any commuting pair $g, h$, we can find a subset of $\left\{\phi\left(\hat{D}_{j}\right)\right\}_{\hat{I}_{\hat{X}}-I_{\text {excep }}}$ which consists of normal crossing divisors, and such that every component of $\left(\mathbb{P}^{2}\right)^{g, h}$ is inside the intersection locus of this subset. Since the change of variables formula applies to every commuting pair individually, this completes the proof.

We also have $\pi^{*} K_{\mathbb{P}^{2} / G}=K_{\bar{Y}}+\sum_{I_{\bar{Y}}} \alpha_{i} \bar{D}_{i}$ for rationals $\alpha_{i}<1$. Since $\psi^{*} K_{\mathbb{P}^{2} / G}=$ $K_{\mathbb{P}^{2}}$, we get $\mu^{*}\left(K_{\bar{Y}}+\sum_{I_{\bar{Y}}} \alpha_{i} \bar{D}_{i}\right)=\mu^{*} \pi^{*} K_{\mathbb{P}^{2} / G}=\phi^{*} K_{\mathbb{P}^{2}}=K_{\hat{X}}+\sum_{I_{\hat{X}}} \beta_{j} \hat{D}_{j}$. 
Lemma 8.3. $\quad \mu_{*} \mathscr{E} l l_{\text {orb }}^{T}\left(\hat{X}, \sum \beta_{j} \hat{D}_{j}, G\right)=\left(\frac{2 \pi i \theta(-z)}{\theta^{\prime}(0)}\right)^{2} \mathscr{E} l l_{T}\left(\bar{Y}, \sum \alpha_{i} \bar{D}_{i}\right)$.

Proof. Since $\mu: \hat{X} \rightarrow \bar{Y}$ is a toroidal morphism, we have $\mu^{*} \Omega\left(\log \sum \alpha_{i} \bar{D}_{i}\right)=$ $\Omega\left(\log \sum \beta_{j} \hat{D}_{j}\right)$. Therefore, by an argument analogous to the proof of Lemma 7.2,

$$
\frac{c_{T}(T \hat{X})}{\mu^{*} c_{T}(T \bar{Y})}=\frac{\prod_{I_{\hat{X}}}\left(1+c_{1}^{T}\left(\hat{D}_{j}\right)\right)}{\prod_{I_{\bar{Y}}}\left(1+\mu^{*} c_{1}^{T}\left(\bar{D}_{i}\right)\right)} .
$$

Following the same argument as in the proof of Theorem 7.1, we are reduced to proving that

$$
\begin{gathered}
\mu_{*} \frac{1}{|G|} \sum_{\substack{g h=h g \\
\hat{X}_{\gamma}^{g, h}}}\left(\Phi_{\hat{X}_{\gamma}^{g, h}}^{T} \prod_{I_{\hat{X}}-I_{\gamma}^{g, h}} \frac{\frac{\hat{D}_{j}}{2 \pi i} \theta\left(\frac{\hat{D}_{j}}{2 \pi i}-\left(-\beta_{j}+1\right) z\right) \theta^{\prime}(0)}{\theta\left(\frac{\hat{D}_{j}}{2 \pi i}\right) \theta\left(-\left(-\beta_{j}+1\right) z\right)}\right. \\
\prod_{I_{\gamma}^{g, h}} \frac{\theta\left(\frac{\hat{D}_{j}}{2 \pi i}+\epsilon_{j}(g)-\epsilon_{j}(h) \tau-\left(-\beta_{j}+1\right) z\right) \frac{\theta^{\prime}(0)}{2 \pi i}}{\theta\left(\frac{\hat{D}_{j}}{2 \pi i}+\epsilon_{j}(g)-\epsilon_{j}(h) \tau\right) \theta\left(-\left(-\beta_{j}+1\right) z\right)} e^{\left.2 \pi i\left(-\beta_{j}+1\right) \epsilon_{j}(h) z\right)} \\
=\prod_{I_{\bar{Y}}} \frac{\frac{\bar{D}_{i}}{2 \pi i} \theta\left(\frac{\bar{D}_{i}}{2 \pi i}-\left(-\alpha_{i}+1\right) z\right) \theta^{\prime}(0)}{\theta\left(\frac{\bar{D}_{i}}{2 \pi i}\right) \theta\left(-\left(-\alpha_{i}+1\right) z\right)} .
\end{gathered}
$$

Let $H \in \mathbb{C} \llbracket \Sigma_{\bar{Y}} \rrbracket$ be the piecewise convergent power series given by

$$
\left.H\right|_{C}=\prod_{i=1}^{\operatorname{dim} C} \frac{\frac{x_{i}^{C}}{2 \pi i} \theta\left(\frac{x_{i}^{C}}{2 \pi i}-\left(-\alpha_{i}+1\right) z\right) \theta^{\prime}(0)}{\theta\left(\frac{x_{i}^{C}}{2 \pi i}\right) \theta\left(-\left(-\alpha_{i}+1\right) z\right)} .
$$

Similarly, let $F_{\gamma}^{g, h} \in \mathbb{C} \llbracket \Sigma_{\hat{X}} \rrbracket$ be defined as in the proof of Theorem 7.1. By Lemma 5.5, we are reduced to proving that

$$
\nu_{*} \frac{1}{|G|} \sum_{g h=h g ; \gamma} F_{\gamma}^{g, h}=H .
$$

Let $C \subset \Sigma_{\bar{Y}}$ be a 2-dimensional cone with lattice $N_{C}$. Let $v^{-1} C$ be the collection of fans $\left\{\Sigma_{C}^{k}\right\}_{k}$. From our construction of $\Sigma_{\hat{X}}$ and $\Sigma_{\bar{Y}}$, the $\Sigma_{C}^{k}$ are isomorphic to a fixed subdivision $\Sigma_{C}$ with lattice $N\left(\Sigma_{C}\right)$. Let $G=N_{C} / N\left(\Sigma_{C}\right)$ and $d$ be the cardinality of the set $\left\{\Sigma_{C}^{k}\right\}$. We clearly have $d \cdot\left|G\left(\Sigma_{C}\right)\right|=|G|$. By the pushforward formula for toroidal morphisms,

$$
\left(v_{*} \frac{1}{|G|} \sum_{g h=h g ; \gamma} F_{\gamma}^{g, h}\right)_{C}
$$


is equal to

$$
\begin{aligned}
\frac{d}{|G|} \sum_{C_{i} \subset \Sigma_{C}} \sum_{g h=h g ; \gamma}\left(F_{\gamma}^{g, h}\right)_{C_{i}} \frac{\prod_{j=1}^{2} x_{j}^{C}}{\prod_{j=1}^{2} x_{j}^{C_{i}}} \\
=\frac{1}{\left|G\left(\Sigma_{C}\right)\right|} \sum_{C_{i} \subset \Sigma_{C}} \sum_{g, h \in G\left(\Sigma_{C}\right)} \\
\quad \times \prod_{j=1}^{2} \frac{\theta\left(\frac{x_{j}^{C_{i}}}{2 \pi i}+\epsilon_{j}(g)-\epsilon_{j}(h) \tau-\left(-\beta_{j}+1\right) z\right) \frac{\theta^{\prime}(0)}{2 \pi i}}{\theta\left(\frac{x_{j}^{C_{i}}}{2 \pi i}+\epsilon_{j}(g)-\epsilon_{j}(h) \tau\right) \theta\left(-\left(-\beta_{j}+1\right) z\right)} e^{2 \pi i\left(-\beta_{j}+1\right) \epsilon_{j}(h) z} \prod_{j=1}^{2} x_{j}^{C} .
\end{aligned}
$$

We wish to show that this last expression is equal to

$$
\prod_{j=1}^{2} \frac{\frac{x_{j}^{C}}{2 \pi i} \theta\left(\frac{x_{j}^{C}}{2 \pi i}-\left(-\alpha_{j}+1\right) z\right) \theta^{\prime}(0)}{\theta\left(\frac{x_{j}^{C}}{2 \pi i}\right) \theta\left(-\left(-\alpha_{j}+1\right) z\right)} .
$$

Since the coefficients $\left\{-\beta_{j}+1\right\}$ are the pullbacks of the coefficients $\left\{-\alpha_{i}+1\right\}$, this identity follows from [Borisov and Libgober 2005, Lemma 8.1].

Now let $p: V \rightarrow \mathbb{C}^{2} / G$ be the $T$-equivariant crepant resolution, i.e., $K_{V}=$ $p^{*} K_{\mathbb{C}^{2} / G}$. By the equivariant factorization theorem for surfaces, we may connect $Y$ to $V$ by a finite sequence of equivariant blow-ups and blow-downs. In other words, we may form the commutative diagram

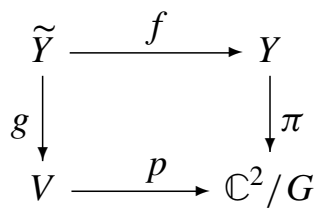

Here $f$ and $g$ are sequences of $T$-equivariant blow-ups. Moreover, we may assume that the blow-ups of $f$ occur at complete intersection points. It is not immediately clear that the same holds true for $g$. However, every blow-up point for $g$ will occur at a $T$-fixed point $p \in D$ for some $D \cong \mathbb{P}^{1}$, with $D T$-invariant. From toric geometry, every such $p$ may be represented as a complete intersection of $T$-invariant divisors in a neighborhood of $D$. By functorial localization, we may therefore assume that $p$ is a complete intersection point when we calculate pushforwards.

Define $\widetilde{D}_{i}$ and $\tilde{\alpha}_{i}$ so that $f^{*}\left(K_{Y}+\sum \alpha_{i} D_{i}\right)=K_{\widetilde{Y}}+\sum \tilde{\alpha}_{i} \widetilde{D}_{i}$. Then

$$
K_{\widetilde{Y}}+\sum \tilde{\alpha}_{i} \widetilde{D}_{i}=f^{*} \pi^{*} K_{\mathbb{C}^{2} / G}=g^{*} K_{V}
$$

By the equivariant change of variables formula and functorial localization, 


$$
\sum_{P \subset V} \int_{P} \frac{\mathscr{E} l l_{T}(V, 0)}{e(P)}=\sum_{F \subset Y} \int_{F} \frac{\mathscr{E} l l_{T}\left(Y, \sum \alpha_{i} D_{i}\right)}{e(F)} .
$$

Here $\{P\}$ and $\{F\}$ run over the fixed components of $V$ and $Y$, respectively. The above formula combined with the previous two lemmas imply the following equivariant elliptic genus analogue of the classical McKay correspondence:

Theorem 8.4. $\quad E l l_{\mathrm{orb}}^{T}\left(\mathbb{C}^{2}, 0, G\right)=\left(\frac{2 \pi i \theta(-z)}{\theta^{\prime}(0)}\right)^{2} E l l_{T}(V, 0)$.

Letting the parameter $z$ go to 0 we get:

Corollary 8.5. The number of irreducible representations of $G$ is $\sum_{P \subset V} \int_{P} e(T P)$. In other words, $e_{\text {orb }}\left(\mathbb{C}^{2}, G\right)=e(V)$.

\section{Relation to Batyrev's stringy Euler number}

Let $X$ be a smooth quasiprojective variety and $D=\sum_{I} a_{i} D_{i}$ an effective divisor with simple normal crossings. Batyrev [1999] defined the stringy Euler number of the pair $(X, D)$ as

$$
e_{\mathrm{str}}(X, D)=\sum_{J \subset I} e\left(D_{J}^{o}\right) \prod_{j \in J} \frac{1}{a_{j}+1} .
$$

Here $D_{J}^{o}=\bigcap_{j \in J} D_{j}-\bigcup_{I-J} D_{i}$. The definition is best understood from the point of view of motivic integration.

Suppose that $X$ has a $T$ action with compact fixed components and that the irreducible components of $D$ are $T$-invariant. Then the equivariant elliptic genus $\operatorname{Ell}_{T}(X,-D)$ is well-defined and it is natural to question how it relates to Batyrev's stringy Euler number. In this section we prove:

Theorem 9.1. Let $X$ and $D$ be as above. Then:

$$
\lim _{y \rightarrow 1} \lim _{q \rightarrow 0}\left(\frac{2 \pi i \theta(-z)}{\theta^{\prime}(0)}\right)^{\operatorname{dim} X} \operatorname{Ell}_{T}(X,-D)=e_{\text {str }}(X, D) .
$$

Proof. We first note that we may rewrite $e_{\text {str }}(X, D)$ in the more convenient form

$$
e_{\mathrm{str}}(X, D)=\sum_{J \subset I} e\left(D_{J}\right) \prod_{j \in J}\left(\frac{1}{a_{j}+1}-1\right) .
$$

Here $D_{J}=\bigcap_{j \in J} D_{j}$. For a quasiprojective $T$-space $V$ with compact fixed components, let $\chi_{-y}^{T}(V)$ denote the equivariant $\chi_{-y}$-genus of $V$; that is,

$$
\chi_{-y}^{T}(V)=\sum_{F \subset V^{T}} \int_{F} \prod_{T F} \frac{f_{i}\left(1-y e^{-f_{i}}\right)}{1-e^{-f_{i}}} \prod_{\nu_{F}} \frac{1-y e^{-n_{j}-w_{j}}}{1-e^{-n_{j}-w_{j}}} .
$$


Here $n_{j}$ are the formal Chern roots of the normal bundle $v_{F}$ to $F$ and $w_{j}$ are the infinitesimal weights of the $T$ action on the fibers of $v_{F}$. It is easy to verify that

$$
\lim _{y \rightarrow 1} \sum_{J \subset I} \chi_{-y}^{T}\left(D_{J}\right) \prod_{j \in J}\left(\frac{y-1}{y^{a_{j}+1}-1}-1\right)=e_{\mathrm{str}}(X, D) .
$$

The above equality essentially follows from the fact that $e(V)$ corresponds to $\sum_{F \subset V^{T}} e(F)$, where $\{F\}$ denotes the collection of fixed components of $V$ (all of which are compact). Thus, we are reduced to identifying

$$
\sum_{J \subset I} \chi_{-y}^{T}\left(D_{J}\right) \prod_{j \in J}\left(\frac{y-1}{y^{a_{j}+1}-1}-1\right)
$$

with a specialization of the equivariant elliptic genus.

Fix an indexing set $J \subset I$ and a fixed component $F$ of $X$ which intersects $D_{J}$ nontrivially. Write $\left\{D_{j}\right\}_{j \in J}$ as the disjoint union of collections $\left\{D_{\alpha}\right\} \cup\left\{D_{\beta}\right\}$, where $D_{\beta}$ denote the divisors which contain $F$. Note that since $F \cap D_{J} \neq \varnothing$, for any of the divisors $D_{\alpha}, D_{\alpha} \cap F$ is a proper subset of $F$.

The collection $\left\{D_{J} \cap F_{i}\right\}_{F_{i} \in \operatorname{Fix}(X)}$ describes a partition of the collection of fixed components of $D_{J}$. Let $P=D_{J} \cap F$. From the preceding discussion we see that $v_{P / F}=\left.\bigoplus_{\alpha} O\left(D_{\alpha}\right)\right|_{P}$.

Consider the integral

$$
\int_{F} \prod_{T F} \frac{f_{i}\left(1-y e^{-f_{i}}\right)}{1-e^{-f_{i}}} \prod_{\nu_{F / X}} \frac{1-y e^{-n_{\ell}-w_{\ell}}}{1-e^{-n_{\ell}-w_{\ell}}} \prod_{\beta} \frac{1-e^{-D_{\beta}-w_{\beta}}}{1-y e^{-D_{\beta}-w_{\beta}}} \prod_{\alpha} \frac{1-e^{-D_{\alpha}}}{1-y e^{-D_{\alpha}}} .
$$

Letting $N=v_{F / X}-\bigoplus_{\beta} O\left(D_{\beta}\right)$, we may rewrite it as

$$
\begin{aligned}
\int_{F} \prod_{T F} \frac{f_{i}\left(1-y e^{-f_{i}}\right)}{1-e^{-f_{i}}} \prod_{N} \frac{1-y e^{-n_{\ell}-w_{\ell}}}{1-e^{-n_{\ell}-w_{\ell}}} & \prod_{\alpha} \frac{1-e^{-D_{\alpha}}}{D_{\alpha}\left(1-y e^{-D_{\alpha}}\right)} \prod_{\alpha} D_{\alpha} \\
= & \int_{P} \prod_{T P} \frac{p_{k}\left(1-y e^{-p_{k}}\right)}{1-e^{-p_{k}}} \prod_{\nu_{P / D_{J}}} \frac{1-y e^{-n_{\ell}-w_{\ell}}}{1-e^{-n_{\ell}-w_{\ell}}} .
\end{aligned}
$$

Summing over all $J \subset I$, we therefore have

$$
\begin{aligned}
\sum_{J \subset I} \chi_{-y}^{T}\left(D_{J}\right) & \prod_{j \in J}\left(\frac{y-1}{y^{a_{j}+1}-1}-1\right) \\
& =\sum_{J, F} \int_{F} \prod_{T F} \frac{f_{i}\left(1-y e^{-f_{i}}\right)}{1-e^{-f_{i}}} \prod_{v_{F / X}} \frac{1-y e^{-n_{\ell}-w_{\ell}}}{1-e^{-n_{\ell}-w_{\ell}}} \prod_{J} \frac{1-e^{-D_{j}-w_{j}}}{1-y e^{-D_{j}-w_{j}}} \frac{y-y^{a_{j}+1}}{y^{a_{j}+1}-1} \\
& =\sum_{F} \int_{F} \prod_{T F} \frac{f_{i}\left(1-y e^{-f_{i}}\right)}{1-e^{-f_{i}}} \prod_{v_{F / X}} \frac{1-y e^{-n_{\ell}-w_{\ell}}}{1-e^{-n_{\ell}-w_{\ell}}} \prod_{I} \frac{1-e^{-D_{i}-w_{i}}}{1-y e^{-D_{i}-w_{i}}} \frac{y-1}{y^{a_{i}+1}-1} .
\end{aligned}
$$



It is easy to see that this last expression equals $\lim _{q \rightarrow 0}\left(\frac{2 \pi i \theta(-z)}{\theta^{\prime}(0)}\right)^{\operatorname{dim} X} E l l_{T}(X,-D)$.
This completes the proof.

\section{Appendix}

Lemma A.1. Let $f: X \rightarrow Y$ be a T-map of smooth compact simply connected complex manifolds. Let $D \subset Y$ be a T-invariant divisor and let $E_{i}$ be T-invariant normal crossing divisors on $X$ such that $f^{*} D=\sum a_{i} E_{i}$ as Cartier divisors. Then for any $\varepsilon$-regular neighborhood $U_{\varepsilon}$ of $D$ there exist generators $\Theta_{E_{i}}^{T}$ for $c_{1}^{T}\left(E_{i}\right)$ and $\Theta_{D}^{T}$ for $c_{1}^{T}(D)$ with the following properties:

(1) $\Theta_{D}^{T}$ has compact support in $U_{\varepsilon}$ and $\Theta_{E_{i}}^{T}$ have compact support in $f^{-1}\left(U_{\varepsilon}\right)$.

(2) $f^{*} \Theta_{D}^{T}=\sum a_{i} \Theta_{E_{i}}^{T}+d_{T}(\eta)$ on the level of forms, where $\eta$ is a T-invariant form with compact support in $U_{\varepsilon}$.

(3) $\Theta_{D}^{T}$ and $\Theta_{E_{i}}^{T}$ represent the extension by zero of the equivariant Thom classes of the varieties $D$ and $E_{i}$.

The only real issue above is to ensure that $\eta$ has compact support in the desired neighborhood.

Proof. We first solve this problem in the nonequivariant category. For $V$ any Cartier divisor, denote by $L_{V}$ the line bundle it induces. Let $U_{\varepsilon}$ be a $T$-invariant tubular neighborhood of $D$ of radius $\varepsilon$. Outside $U_{\varepsilon / 2}$, the constant function 1 is a section of $L_{D}$. Define a metric $h_{\text {far }}$ in this region by $h_{\text {far }}=\|1\|^{2} \equiv 1$. Let $h_{\text {near }}$ be a metric inside $U_{\varepsilon}$. Piece the two metrics into a global metric $h$ on $L_{D}$ using a partition of unity. The first Chern class of $L_{D}$ is then represented by the form $\Theta_{D}=\frac{i}{2 \pi} \bar{\partial} \partial \log h$. This form clearly has compact support in $U_{\varepsilon}$.

Let $U_{\varepsilon_{i}}$ be tubular neighborhoods of $E_{i}$. Choose $\varepsilon_{i}$ small enough so that each of these neighborhoods is contained in $f^{-1} U_{\varepsilon}$. Define metrics $h_{i}$ on $E_{i}$ in a manner analogous to the above construction of $h$. Clearly the forms $\Theta_{E_{i}}=\frac{i}{2 \pi} \bar{\partial} \partial \log h_{i}$ have compact support in $U_{\varepsilon_{i}}$ and represent the first Chern classes of $L_{E_{i}}$.

We have two natural choices for a metric on $f^{*} L_{D}$, namely $f^{*} h$ and $h_{1}^{a_{1}} \cdots h_{k}^{a_{k}}$. Choose a smooth nonzero function $\varphi$ so that $f^{*} h=\varphi h_{1}^{a_{1}} \cdots h_{k}^{a_{k}}$. Notice that $\varphi \equiv 1$ outside $f^{-1} U_{\varepsilon}$. We have

$$
\bar{\partial} \partial \log f^{*} h=\bar{\partial} \partial \log \varphi+\sum_{i} a_{i} \bar{\partial} \partial \log h_{i} .
$$

But this implies that $f^{*} \Theta_{D}=\sum_{i} a_{i} \Theta_{E_{i}}+\frac{i}{2 \pi} \bar{\partial} \partial \log \varphi$. If we let $d^{c}=\frac{i}{4 \pi}(\bar{\partial}-\partial)$, we may write this last equation as

$$
f^{*} \Theta_{D}=\sum_{i} a_{i} \Theta_{E_{i}}-d d^{c} \log \varphi .
$$


The form $\eta=-d^{c} \log \varphi$ clearly has compact support in $f^{-1} U_{\varepsilon}$. It remains to argue that $\Theta_{D}$ and $\Theta_{E_{i}}$ represent the Thom classes of $D$ and $E_{i}$. It is a standard fact that these classes are Poincaré duals to their respective divisors. If a divisor is homologously nontrivial, then clearly its Thom classes coincides with its Poincaré dual. If a divisor is homologously trivial, then it must follow that the extension by zero of its Thom class is trivial. Either way this implies that the above classes represent the extension by zero of the Thom classes of their respective divisors. This completes the nonequivariant portion of the proof.

By averaging over the group $T$, we may assume that all the forms above are $T$ invariant. For notational simplicity, let us assume that $T=S^{1}$. Let $V$ be the vector field on $X$ induced by the $T$-action. Let $g_{i}$ be the functions compactly supported in $f^{-1} U_{\varepsilon}$ which satisfy the moment map equation $i_{V} \Theta_{E_{i}}=d g_{i}$. Similarly, let $W$ be the vector field on $Y$ defined by the $T$-action and define $g$ so that it satisfies $i_{V} \Theta_{D}=$ $d g$ and has support inside $U_{\varepsilon}$. Note that since $f$ is $T$-equivariant, $i_{V} f^{*} \Theta_{D}=$ $f^{*} i_{W} \Theta_{D}=f^{*} d g$. We then have $d(g \circ f)=\sum_{i} a_{i} d g_{i}+i_{V} d \eta=\sum_{i} a_{i} d g_{i}-d i_{V} \eta$. Hence $g \circ f=\sum_{i} a_{i} g_{i}-i_{V} \eta$. But this implies that

$$
f^{*}\left(\Theta_{D}+g\right)=\sum_{i} a_{i}\left(\Theta_{E_{i}}+g_{i}\right)+\left(d-i_{V}\right) \eta .
$$

But this is precisely the relation we wish to in the equivariant cohomology.

Lemma A.2 (Excess intersection formula). Let $X$ be a smooth compact variety with irreducible normal crossing divisors $D_{1}, \ldots, D_{k}$. For $I \subset\{1, \ldots, k\}$ denote by $X_{I, j}$ the $j$-th connected component of $\bigcap_{I} D_{i}$ and by $\Phi_{I, j}$ its Thom class. Fix irreducible subvarieties $X_{I_{1}, j_{1}}$ and $X_{I_{2}, j_{2}}$. For $I_{0}=I_{1} \cup I_{2}$, let $X_{I_{0}, j}$ be the irreducible components of $X_{I_{1}, j_{1}} \cap X_{I_{2}, j_{2}}$. Then

$$
\Phi_{I_{1}, j_{1}} \wedge \Phi_{I_{2}, j_{2}}=\sum_{I_{0}, j} \Phi_{I_{0}, j} \prod_{I_{1} \cap I_{2}} \Phi_{i}
$$

Proof. Let $N_{I, j}$ be tubular neighborhoods of $X_{I, j}$ which are disjoint for each indexing set $I$ and which satisfy $N_{I, j} \subset N_{I^{\prime}, j^{\prime}}$ for $X_{I, j} \subset X_{I^{\prime}, j^{\prime}}$. If we choose $\Phi_{i}$ to have compact support in a sufficiently small tubular neighborhood of $D_{i}$, then $\prod_{I} \Phi_{i}$ will have compact support in $\bigsqcup_{j} N_{I, j}$. The extension by zero of $\left.\left(\prod_{I} \Phi_{i}\right)\right|_{N_{I, j}}$ will represent the Thom class of $X_{I, j}$ (see [Bott and Tu 1982]). We may also ensure that $\Phi_{I_{1}, j_{1}} \wedge \Phi_{I_{2}, j_{2}}$ has compact support in $\bigsqcup_{j} N_{I_{0}, j}$.

$$
\begin{aligned}
\Phi_{I_{1}, j_{1}} \wedge \Phi_{I_{2}, j_{2}} & =\left.\sum_{I_{0}, j}\left(\prod_{I_{1}} \Phi_{i} \prod_{I_{2}} \Phi_{i}\right)\right|_{N_{I_{0}, j}}=\left.\sum_{I_{0}, j}\left(\prod_{I_{0}} \Phi_{i} \prod_{I_{1} \cap I_{2}} \Phi_{i}\right)\right|_{N_{I_{0}, j}} \\
& =\left.\sum_{I_{0}, j}\left(\prod_{I_{0}} \Phi_{i}\right)\right|_{N_{I_{0}, j}} \prod_{I_{1} \cap I_{2}} \Phi_{i} .
\end{aligned}
$$


This yields the desired formula.

This proof clearly extends to the equivariant category.

\title{
References
}

[Atiyah and Bott 1984] M. F. Atiyah and R. Bott, "The moment map and equivariant cohomology", Topology 23:1 (1984), 1-28. MR 85e:58041 Zbl 0521.58025

[Batyrev 1999] V. V. Batyrev, "Non-Archimedean integrals and stringy Euler numbers of log-terminal pairs”, J. Eur. Math. Soc. 1:1 (1999), 5-33. MR 2001j:14018 Zbl 0943.14004

[Borisov and Gunnells 2001] L. A. Borisov and P. E. Gunnells, "Toric varieties and modular forms", Invent. Math. 144:2 (2001), 297-325. MR 2002g:11053 Zbl 1039.11022

[Borisov and Libgober 2005] L. Borisov and A. Libgober, "McKay correspondence for elliptic genera”, Ann. of Math. (2) 161:3 (2005), 1521-1569. MR 2008b:58030 Zbl 05004657

[Bott and Tu 1982] R. Bott and L. W. Tu, Differential forms in algebraic topology, Graduate Texts in Mathematics 82, Springer, New York, 1982. MR 83i:57016 Zbl 0496.55001

[Fulton 1993] W. Fulton, Introduction to toric varieties, Annals of Mathematics Studies 131, Princeton University Press, Princeton, NJ, 1993. MR 94g:14028 Zbl 0813.14039

[Hattori 2006] A. Hattori, "Elliptic genera, torus orbifolds and multi-fans, II", Internat. J. Math. 17:6 (2006), 707-735. MR 2007d:58037 Zbl 1101.57014

[Lian et al. 1999a] B. H. Lian, K. Liu, and S.-T. Yau, "Mirror principle, I", pp. 405-454 in Surveys in differential geometry 5: differential geometry inspired by string theory, International Press, Boston, 1999. MR 1772275 Zbl 0999.14010

[Lian et al. 1999b] B. H. Lian, K. Liu, and S.-T. Yau, "Mirror principle. II", pp. 455-509 in Surveys in differential geometry 5: differential geometry inspired by string theory, International Press, Boston, 1999. MR 1772276 Zbl 0999.14011

[Liu 1996] K. Liu, “On elliptic genera and theta-functions”, Topology 35:3 (1996), 617-640. MR 97h:58149 Zbl 0858.57034

[Waelder 2007] R. Waelder, "Equivariant elliptic genera and local McKay correspondences", preprint, 2007. arXiv math.AG/0701336

[Włodarczyk 2003] J. Włodarczyk, “Toroidal varieties and the weak factorization theorem”, Invent. Math. 154:2 (2003), 223-331. MR 2004m:14113 Zbl 02021105

Received September 28, 2007.

\author{
ROBERT WAELDER \\ MATHEMATICS DEPARTMENT \\ UNIVERSITY OF CALIFORNIA \\ Box 951555 \\ Los ANGELES, CA 90095-1555 \\ UNITED STATES \\ rwaelder@math.ucla.edu
}

